AFRL-IF-RS-TR-2003-284

Final Technical Report

November 2003

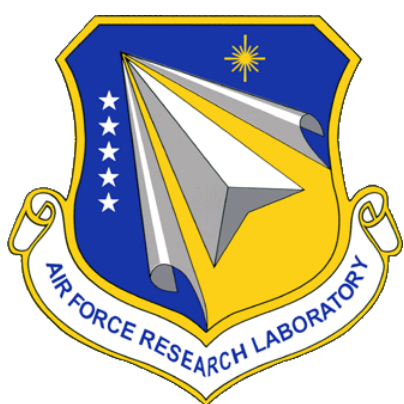

\title{
GLOBAL INFORMATION ENTERPRISE SIMULATION LABORATORY (GIESIM LAB)
}

Prediction Systems, Inc.

APPROVED FOR PUBLIC RELEASE; DISTRIBUTION UNLIMITED.

AIR FORCE RESEARCH LABORATORY

INFORMATION DIRECTORATE

ROME RESEARCH SITE

ROME, NEW YORK 


\section{STINFO FINAL REPORT}

This report has been reviewed by the Air Force Research Laboratory, Information Directorate, Public Affairs Office (IFOIPA) and is releasable to the National Technical Information Service (NTIS). At NTIS it will be releasable to the general public, including foreign nations.

AFRL-IF-RS-TR-2003-284 has been reviewed and is approved for publication

\begin{tabular}{cc} 
APPROVED: & \multicolumn{1}{c}{$/ \mathrm{s} /$} \\
& RICHARD N. SMITH \\
& Project Engineer
\end{tabular}

FOR THE DIRECTOR:

/s/

WARREN H. DEBANY, JR.

Technical Advisor

Information Grid Division

Information Directorate 


\begin{tabular}{|c|c|c|c|c|c|}
\hline \multicolumn{4}{|c|}{ REPORT DOCUMENTATION PAGE } & \multicolumn{2}{|c|}{$\begin{array}{l}\text { Form Approved } \\
\text { OMB No. 074-0188 }\end{array}$} \\
\hline \multicolumn{6}{|c|}{ 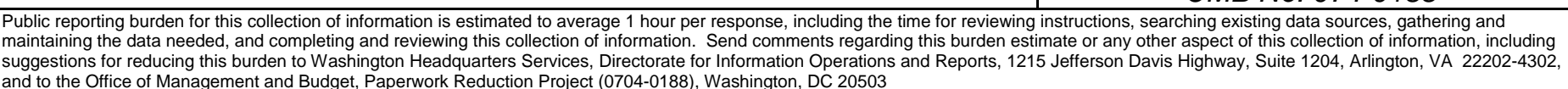 } \\
\hline \multicolumn{2}{|c|}{ 1. AGENCY USE ONLY (Leave blank) } & $\begin{array}{l}\text { 2. REPORT DATE } \\
\text { NOVEMBER } 2003\end{array}$ & \multicolumn{3}{|c|}{$\begin{array}{l}\text { 3. REPORT TYPE AND DATES COVERED } \\
\text { Final } \\
\end{array}$} \\
\hline \multicolumn{4}{|c|}{$\begin{array}{l}\text { 4. TITLE AND SUBTITLE } \\
\text { GLOBAL INFORMATION ENTERPRISE SIMULATION LABORATORY } \\
\text { (GIESim Lab) }\end{array}$} & \multirow{2}{*}{\multicolumn{2}{|c|}{$\begin{array}{l}\text { 5. FUNDING NUMBERS } \\
C \quad-\text { F30602-03-C-0080 } \\
\text { PE - 62702F } \\
\text { PR - GIEP } \\
\text { TA - SI } \\
\text { WU - } 03\end{array}$}} \\
\hline \multicolumn{4}{|l|}{ 6. AUTHOR(S) } & & \\
\hline \multicolumn{4}{|c|}{$\begin{array}{l}\text { 7. PERFORMING ORGANIZATION NAME(S) AND ADDRESS(ES) } \\
\text { Prediction Systems, Inc. } \\
309 \text { Morris Avenue } \\
\text { Spring Lake NJ } 07762\end{array}$} & $\begin{array}{l}\text { 8. PERFOF } \\
\text { REPOR } \\
\text { N/A }\end{array}$ & $\begin{array}{l}\text { NG ORGANIZATION } \\
\text { JMBER }\end{array}$ \\
\hline \multicolumn{4}{|c|}{$\begin{array}{l}\text { 9. SPONSORING / MONITORING AGENCY NAME(S) AND ADDRESS(ES) } \\
\text { AFRL/IFGC } \\
525 \text { Brooks Road } \\
\text { Rome NY 13441-4505 }\end{array}$} & \multicolumn{2}{|c|}{$\begin{array}{l}\text { 10. SPONSORING I MONITORING } \\
\text { AGENCY REPORT NUMBER } \\
\text { AFRL-IF-RS-TR-2003-284 }\end{array}$} \\
\hline \multicolumn{6}{|l|}{ 11. SUPPLEMENTARY NOTES } \\
\hline \multicolumn{5}{|c|}{$\begin{array}{l}\text { 12a. DISTRIBUTION / AVAILABILITY STATEMENT } \\
\text { Approved for Public Release; Distribution Unlimited }\end{array}$} & 12b. DISTRIBUTION CODE \\
\hline \multicolumn{6}{|c|}{$\begin{array}{l}\text { 13. ABSTRACT (Maximum } 200 \text { Words) } \\
\text { The GIESim project is a simulation development program to define, design and implement a Modeling and Simulation } \\
\text { (M\&S) framework for the GIE. Within the GIESim framework, users will be able to execute, via a common interface, } \\
\text { multiple communications and network M\&S tools to most effectively and efficiently analyze candidate communications } \\
\text { architectures and technologies. The GIESim will interface with other M\&S tools (e.g., force-level simulations and } \\
\text { detailed hardware system models) to provide the appropriate level of M\&S fidelity and processing speed for the broad } \\
\text { spectrum of M\&S tasks. The GIESim user base will span from advanced technology researchers to communications } \\
\text { network architects to mission planners. }\end{array}$} \\
\hline \multirow{2}{*}{\multicolumn{5}{|c|}{$\begin{array}{l}\text { 14. SUBJECT TERMS } \\
\text { Modeling and Simulation, Distributed Simulation, Communications Simulation }\end{array}$}} & $\begin{array}{l}\text { 15. NUMBER OF PAGES } \\
43\end{array}$ \\
\hline & & & & & 16. PRICE CODE \\
\hline \multirow{2}{*}{$\begin{array}{l}\text { 17. SECURITY CLASSIFICATION } \\
\text { OF REPORT } \\
\text { UNCLASSIFIED }\end{array}$} & \multirow{2}{*}{\multicolumn{2}{|c|}{$\begin{array}{l}\text { 18. SECURITY CLASSIFICATION } \\
\text { OF THIS PAGE }\end{array}$}} & \multicolumn{2}{|c|}{$\begin{array}{l}\text { 19. SECURITY CLASSIFICATION } \\
\text { OF ABSTRACT }\end{array}$} & 20. LIMITATION OF ABSTRACT \\
\hline & & & & FIED & UL \\
\hline \multicolumn{4}{|l|}{ NSN 7540-01-280-5500 } & & $\begin{array}{l}\text { Idard Form } 298 \text { (Rev. 2-89) } \\
\text { ribed by ANSI Std. Z39-18 } \\
02\end{array}$ \\
\hline
\end{tabular}




\section{TABLE OF CONTENTS}

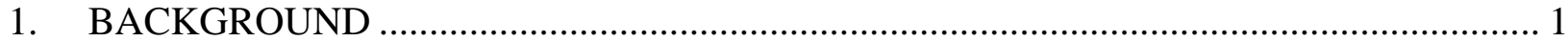

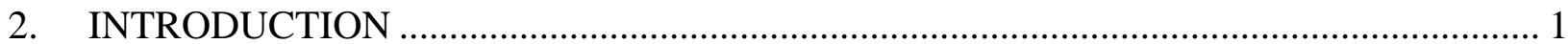

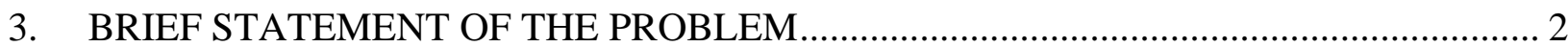

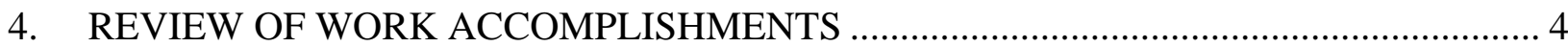

4.1 BACKGROUND ON THE MULTI-SIMULATION DEMONSTRATION ……........... 4

4.2 OVERVIEW OF PSI DEVELOPMENTS ................................................................ 8

4.3 HLA INTERFACE AND TEST_DRV DEVELOPMENT …….................................. 10

4.4 JTIDS SIMULATION DEVELOPMENTS FOR GIESIM ....................................... 13

4.5 SAT_COM SIMULATION DEVELOPMENTS FOR GIESIM.................................. 16

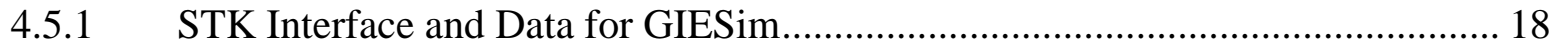

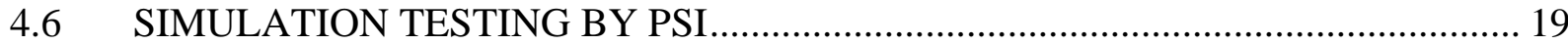

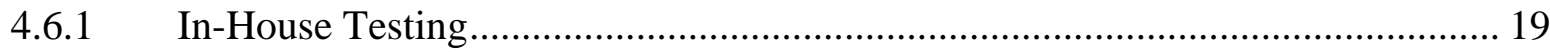

4.6.2 Internet HLA Interoperability Tests.................................................................... 20

4.6.3 Dry Run Interoperability Testing at AFRL Rome ............................................... 20

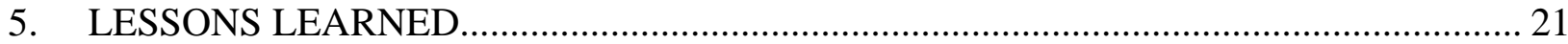

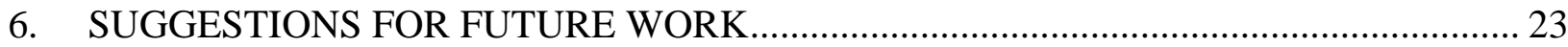

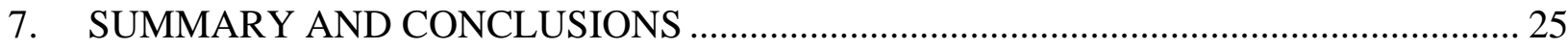

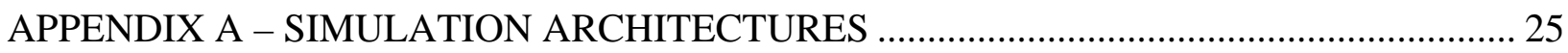

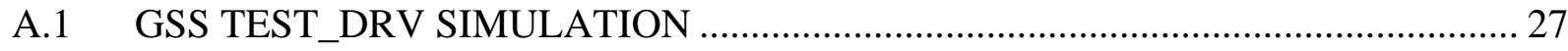

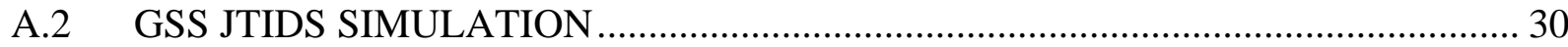

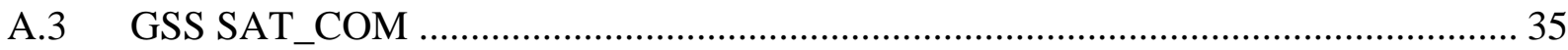




\section{LIST OF FIGURES}

Figure 1 - DTIG Multi-Simulation Demo (DMSD) Scenario................................................ 4

Figure 2 - Physical Architecture of the GIESim Demo ...................................................... 5

Figure 3 - GSS Simulation GIESim Elements and HLA IDs ............................................ 7

Figure 4 - PSI Work Accomplished for GIESim SAB Demo ............................................... 8

Figure 5 - Main GUI of PSI's HLA Test Driver - TEST_DRV .......................................... 11

Figure 6 - GSS Panels for Pub/Sub of HLA Interactions ................................................. 12

Figure 7 - JTIDS Simulation with GIESim Demo Modifications ........................................ 14

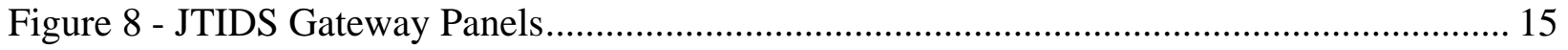

Figure 9 - JTIDS Gateway Buffer Size Panel.................................................................... 15

Figure 10 - SAT_COM World View ............................................................................... 17

Figure 11 - SAT_COM Korean Terrain View ................................................................... 18

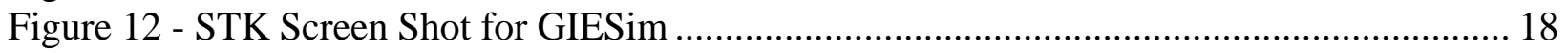

Figure 13 - PSI In-House Test Configuration for GIESim Work ............................................ 19

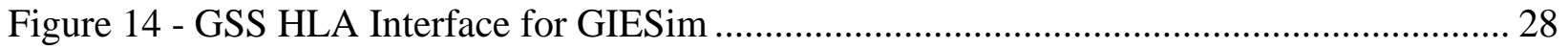

Figure 15 - PSI HLA Test Driver - TEST_DRV ............................................................. 29

Figure 16 - GSS JTIDS Simulation Architecture ............................................................. 31

Figure 17 - Detailed Architecture of GIESim Interfaces in JTIDS ........................................ 32

Figure 18 - AOC_GW Model in JTIDS with AOC Panel Shown ............................................ 33

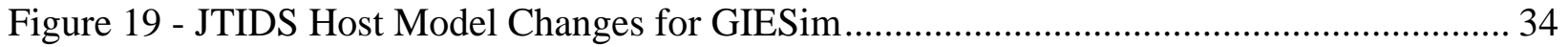

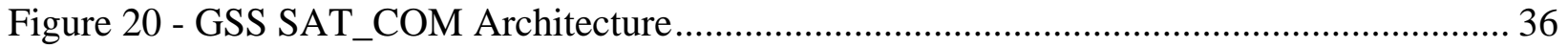

Figure 21 - Detailed Models added to SAT_COM for GIESim ................................................ 37

Figure 22 - SAT_COM STK Interface Control Model.......................................................... 38

\section{LIST OF TABLES}

Table 1- JTIDS Operational Nets Defined for GIESim SAB Demo ....................................... 13 


\section{BACKGROUND}

As more sophisticated systems are required to combat rapidly growing threats, the Air Force faces greater challenges in getting these systems from an R\&D concept to production. A significant challenge is dealing with the level of design complexity imposed by these systems. As software replaces hardware to accommodate the smarts built into a system to make it user friendly, the dimensionality of the design problem grows exponentially. As systems get smarter, they depend upon more information to operate. This implies interfaces with other systems in the Global Information Enterprise (GIE) - to request and obtain information, and to publish and subscribe data to meet shrinking time requirements. At the same time, breadboards and brassboards are becoming very expensive to build. Fortunately, they are becoming less important due to the software nature of these systems. Much of the guts of a system today lay in huge sets of imbedded algorithms in general purpose processors that provide the smarts. Testing these algorithms with all of the external interfaces they must support is becoming most difficult and expensive. To meet these challenges, a new approach to designing and testing these complex systems has evolved. This approach uses simulation.

\section{INTRODUCTION}

This is the Final Report from PSI on our participation in the second round of effort on the formation of the GIESim Laboratory. Since the beginning of this effort, the goals of the GIESim effort have remained the same. GIESim must be capable of predicting the end-to-end performance and survivability of globally distributed information exchange and management applications, such as the Joint Battlespace Infosphere (JBI), Deployable Theater Information Grid (DTIG), and Information For Global Reach (IFGR). It is aimed at providing a powerful and dynamic generic modeling and simulation framework as a baseline for continuing simulations of future instantiations of JBI and other applications.

In PSI's view, such a simulation facility can be used to support development and testing of many systems in various stages of their evolution, e.g., requirements analysis, system design, interface design, system testing, and support for design and test of system upgrades. In effect, GIESim can provide a laboratory for defining, designing, and testing complex communications and information networking components of the GIE. It can support live field testing by helping to plan tests as well as augment and extend test capabilities beyond what is achievable with the actual hardware. Given that simulations have been validated, they can support the interpolation and extrapolation of limited amounts of test data, a major factor in system evaluations and decisions.

This Final Report describes activities, work done, and results of building the multi-simulation demonstration for the SAB review. This report also presents an overview of the simulation architectures of the GSS-based simulations and highlights the changes made to support the SAB GIESim multi-simulation demonstration. This Final Report also presents an overview of the "lessons learned" in the development of the GIESim demonstration, and suggests future activities and directions to ensure the successful evolution and application of the GIESim Laboratory. 


\section{BRIEF STATEMENT OF THE PROBLEM}

The vision of the Global Information Enterprise (GIE) is to move, process, manage, and protect the C2ISR information that supports the functions of Global Awareness and Dynamic Planning and Execution. The mission of GIE is to link aerospace assets intheater and globally, to integrate C2 \& ISR networks, to defend critical information systems from cyber attack, and to develop new information processing and management techniques.

Most large-scale force level simulations assume perfect communications. This can lead to significant limitations and inaccuracies in the results obtained from running these simulations. Tools are needed to bridge these communications modeling gaps.

The GIESim project is a simulation development program to define, design and implement a Modeling and Simulation (M\&S) framework for the GIE. Within the GIESim framework, users will be able to execute, via a common interface, multiple communications and network M\&S tools to most effectively and efficiently analyze candidate communications architectures and technologies. The GIESim will interface with other M\&S tools (e.g., force-level simulations and detailed hardware system models) to provide the appropriate level of M\&S fidelity and processing speed for the broad spectrum of M\&S tasks. The GIESim user base will span from advanced technology researchers to communications network architects to mission planners.

A predictive framework needs to be established to ensure that battlespace information platforms are supported with required communications technologies. This framework, embodied in the GIESim lab, must be capable of predicting endto-end performance and survivability of globally distributed information exchange and management applications, such as the Joint Battlespace Infosphere (JBI).

What will it take to ensure the success of GIESim? What does success imply? Based upon prior experiences in this area, PSI offers the following thoughts. One can envision a simulation laboratory where program managers sign up to make use of the facilities. They are motivated because they can save precious time and money getting answers to complex technical questions. They can use the facility to demonstrate the level of operational capability of systems under test. The results obtained can be validated by targeted testing and in-depth analysis. Most importantly, this laboratory evolves to be a reliable proving ground to support decisions on fielding systems. It also provides a repository for knowledge of what it takes to ensure successful use of R\&D funding.

Given that the above vision is desired, what will it take to ensure its success? Success will be measured in the eyes of the beholders - the users and the decision makers for funding. If the JBI program is the important first user, what is needed to ensure its successful use? What is the JBI program looking for in terms of a simulation environment? What investments are needed to ensure JBI can make good use of GIESim? Can these investments be justified for JBI alone? If not, how can they be leveraged with other programs. What are the milestones, time frames and resources required to ensure the success of GIESim in the long run? PSI is committed to help answer these questions.

For 2003, the GIESim AFRL/IFGC leadership team has set one goal for the GIESim Lab: design and develop an initial proof of concept, heterogeneous multi-simulation environment targeted for demonstration to the AFRL Scientific Advisory Board (SAB) in the fall. The scenarios for this 
demonstration will involve selected sub-components of the Deployable Theater Information Grid (DTIG). The General Simulation System (GSS) from PSI has been identified as a critical part in this demonstration system. 


\section{REVIEW OF WORK ACCOMPLISHMENTS}

This section presents background on the SAB multi-simulation demonstration, an overview of PSI work, then presents details on the work done by PSI for the demonstration.

\subsection{BACKGROUND ON THE MULTI-SIMULATION DEMONSTRATION}

For round two (FY03) of the evolution of GIESim , the GIESim Leadership requested that several teams cooperate to develop a multi-simulation demonstration of the GIESim concept of integrating diverse simulations (primarily of communications) by building a framework with underlying HLA connectivity. This multi-simulation was to "model” aspects of the Deployable Theater Information Grid (DTIG). PSI was one of the companies chosen to participate in the creation of this DTIG Multi-Simulation Demonstration (DMSD). PSI proposed to use it's high-fidelity simulation of JTIDS/Link-16 for a central part of the communications modeling for DMSD, and also proposed to use it's SAT_COM simulation to model satellite communications. PSI also offered a stretch goal of interfacing SAT_COM to the Satellite Tool Kit (STK) from Analytical Graphics to obtain satellite orbits. These proposals were accepted. In addition, the rich, interactive graphics capabilities of the GSS Run-Time Graphics (RTG) would serve as a central means to visualize key behavior and metrics in the demonstration. The DMSD is aimed at the SAB Review now scheduled for November '03. Greg Hadynski of DTIG reviewed the DMSD descriptions and agreed that DMSD represented a piece of the "as-is” DTIG situation.

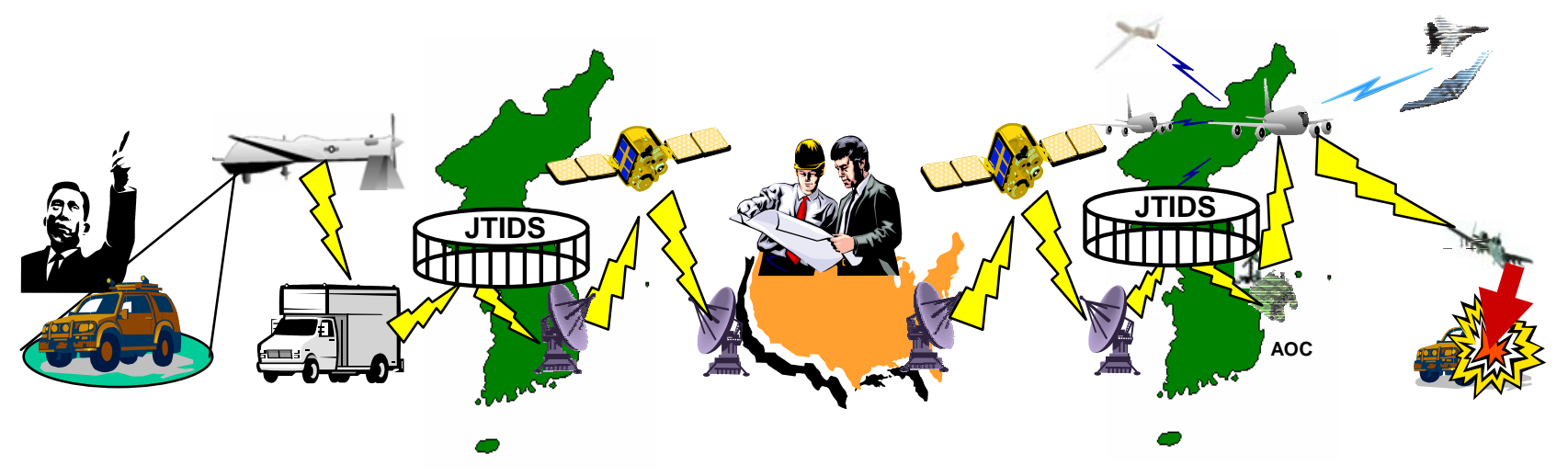

Figure 1 - DTIG Multi-Simulation Demo (DMSD) Scenario ${ }^{1}$

DMSD would involve a scenario in which different simulation components compute their own latency and add it to the accumulating latency as a large UAV Image message works it's way through various communications and information path-ways. Figure 1 shows an illustration for the scenario created for the DMSD by the GIESim team.

The physical architecture of the GIESim DMSD simulations is shown in Figure 2. All simulations typically run on separate PCs and are connected to a LAN that is typically running on a separate Hub to isolate the GIESim DMSD from other LAN traffic. The HLA Run Time Infrastructure (RTI) can run on any of the simulation PCs or a separate PC. In Figure 2, IP refers to the use of TCP/IP sockets, and HLA refers to HLA connections on the LAN. Note that GSS has built-in support for TCP/IP sockets by virtue of IP Channel Resources. This GSS capability is discussed later.

\footnotetext{
${ }^{1}$ From a poster developed by SRC with the GIESim team for the SAB Review
} 


\section{Physical Architecture of GIESim SAB Simulation Demo}

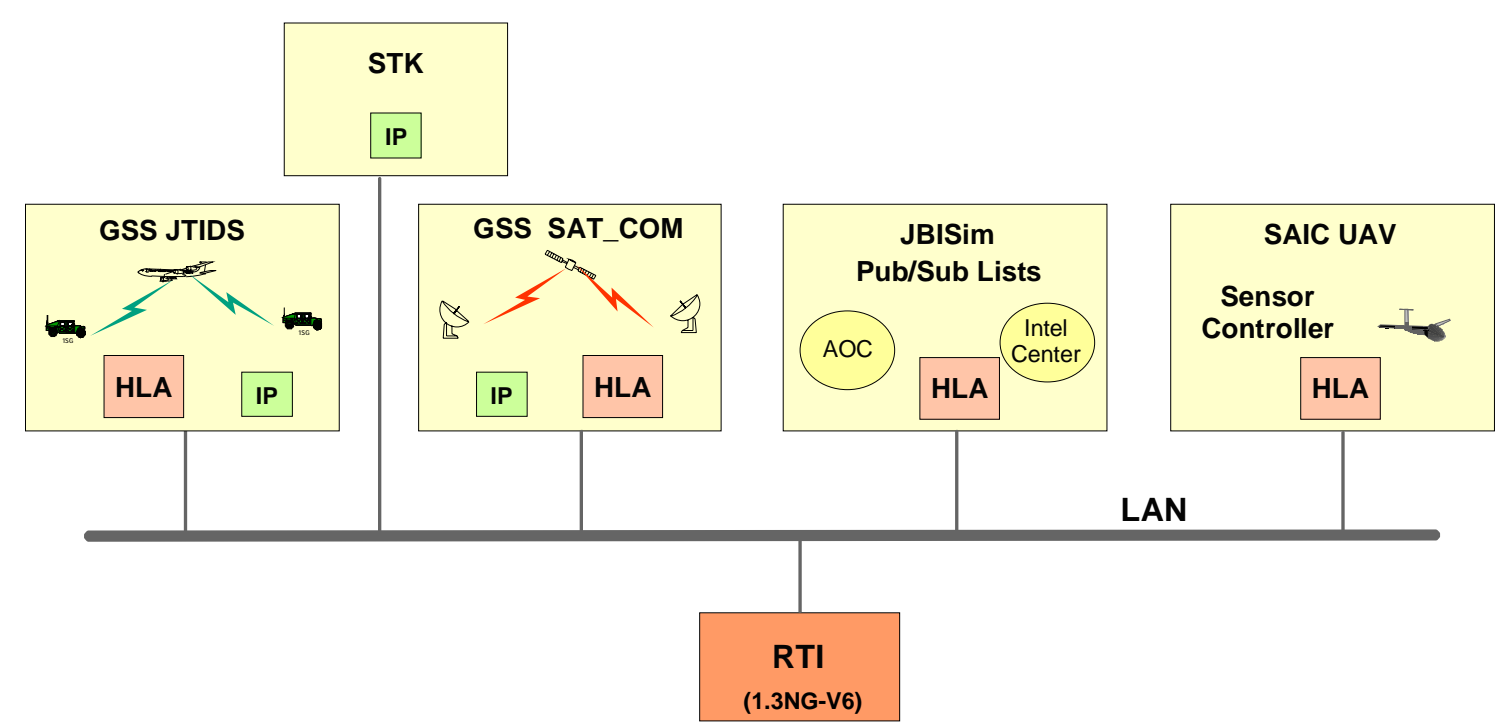

Figure 2 - Physical Architecture of the GIESim Demo

This scenario created for the DMSD by the GIESim team has the following sequence of events :

1. A Predator UAV being modeled by SAIC using OPNET would fly over Korea looking for targets. This simulation would publish HLA interactions that would "fly" the UAV in the visual space of the JTIDS simulation.

2. At some point, the Predator UAV would capture an image of a Time Critical Target (TCT) on the ground in a Korean theater of operation. When this happens, an extra field in the HLA interaction will cause the UAV to "flash" in the JTIDS simulation to indicate image "capture".

3. The Predator UAV would send the image to an Air Operation Center (AOC) component in Korea via HLA interactions to JBISim, which is a simulation of the Joint Battlespace Infosphere.

4. Modeled entities in JBISim would then send the image via HLA to the JTIDS gateway for transmission over JTIDS via the GSS JTIDS simulation.

5. The message sent through the JTIDS network would be received by another gateway in Korea for transmission over a satellite network to the Continental US (CONUS). The JTIDS simulation gateway would send the message to the SAT_COM simulation using a GSS IP Channel interface.

6. The SAT_COM simulation Korean gateway would receive the message from the IP Channel interface and send it to the satellite uplink. This satellite network was modeled in GSS with satellite orbits obtained from STK.

7. At the SAT_COM satellite down-link gateway in CONUS, the received message would be published via HLA for arrival at another component of JBISim.

8. JBISim would model CONUS entities that eventually generate an annotated version of the original image to send back to Korea to direct a strike on the ground TCT. An HLA interaction would be 
published to send the image to the CONUS gateway of the SAT_COM simulation for uplink to Korea.

9. The SAT_COM downlink gateway in Korea would receive the message and send it to the JTIDS simulation via the GSS IP Channel interface.

10. When received by the JTIDS simulation, the gateway would send the message over JTIDS to the gateway associated with the AOC.

11. The receiving gateway in the JTIDS simulation would then publish an HLA interaction to send the message to modeled entities within JBISim.

12. Modeled entities in JBISim would exchange messages with each other.

13. Eventually a strike command is issued via an HLA interaction to a strike platform being modeled in the JTIDS simulation.

14. When the HLA-based strike message is received by the JTIDS simulation it is sent to the strike platform over a JTIDS Net defined for this purpose.

15. When the strike platform receives the message it would send an acknowledgement.

16. The JTIDS gateway would receive the acknowledgement and publish an HLA interaction to send it to JBISim.

A view of the GSS-based simulation components is shown in Figure 3. This figure shows the HLA Entity IDs defined for the GIESim DMSD, and the HLA and TCP/IP socket connectivity of the PSI simulations into the GIESim DMSD. 
Also shown in Figure 3 are new icons that were created for the new GIESim gateways in JTIDS and SAT_COM. In addition to creating gateway icons for the JTIDS simulation, the simulation was modified to "recognize" these icons as having JTIDS radios. This is important for the demo for two reasons. First, as radio equipment icons, the AOC_GW and JTIDS_SAT_GW icons can serve as sources and destination for JTIDS Operational Nets. This capability is needed to support the JTIDS networking for the GIESim messages. Second, as radio icons, the JTIDS simulation can graphically show the JTIDS Links and Nets to and from AOC_GW and JTIDS_SAT_GW. Visualization of the GIESim JTIDS networking components for the DMSD is extremely valuable, and makes for a far richer demonstration.

A new Predator UAV icon was also created for JTIDS. An external simulation provided by SAIC "flys" the Predator icon over the Korean terrain in the JTIDS simulation. An icon for an AOC was also added. The AOC icon is shown in Figure 7 in a later section. As the UAV flies, it is connected to the AOC icon by a line that represents a different communication channel. The dynamically moving UAV in the JTIDS simulation makes for a much more "integrated" and interesting demonstration.

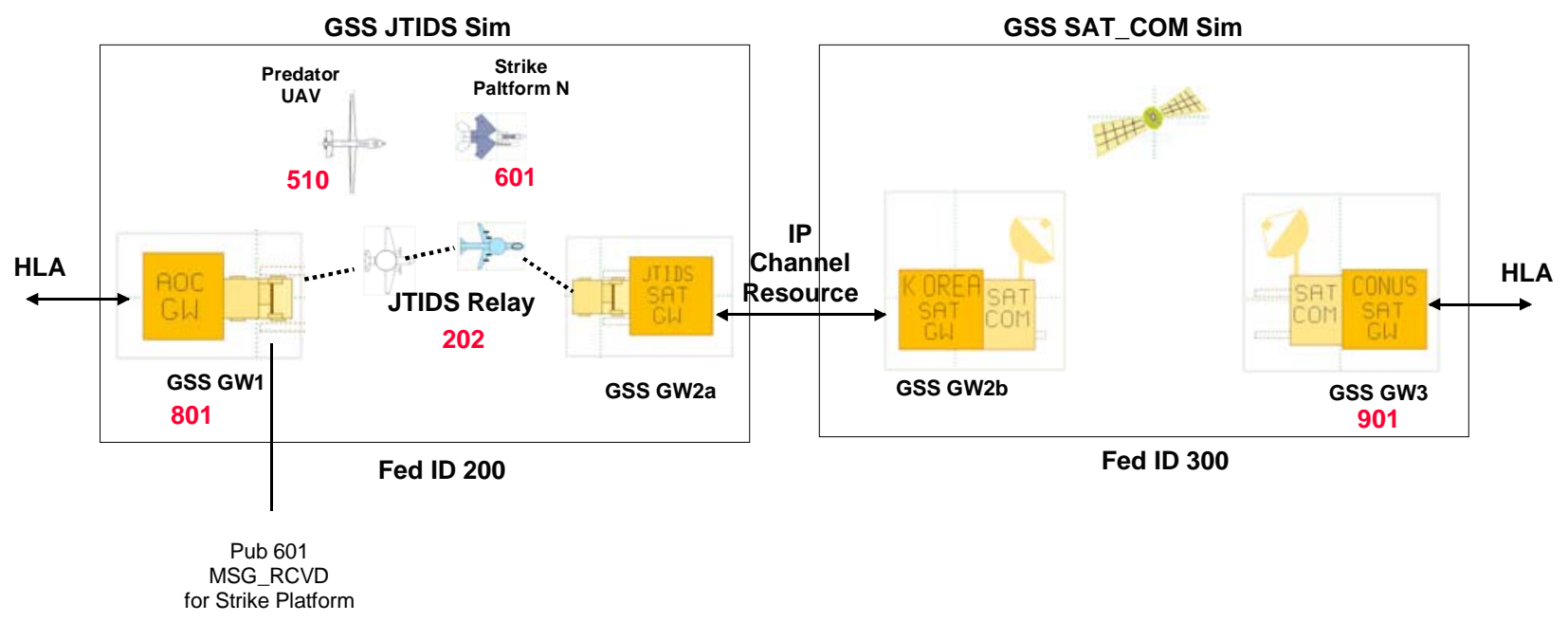

Figure 3 - GSS Simulation GIESim Elements and HLA IDs 


\subsection{OVERVIEW OF PSI DEVELOPMENTS}

Figure 4 shows an illustration of the work items accomplished by PSI for the GIESim SAB Demo and the different components within each simulation in support of GIESim.

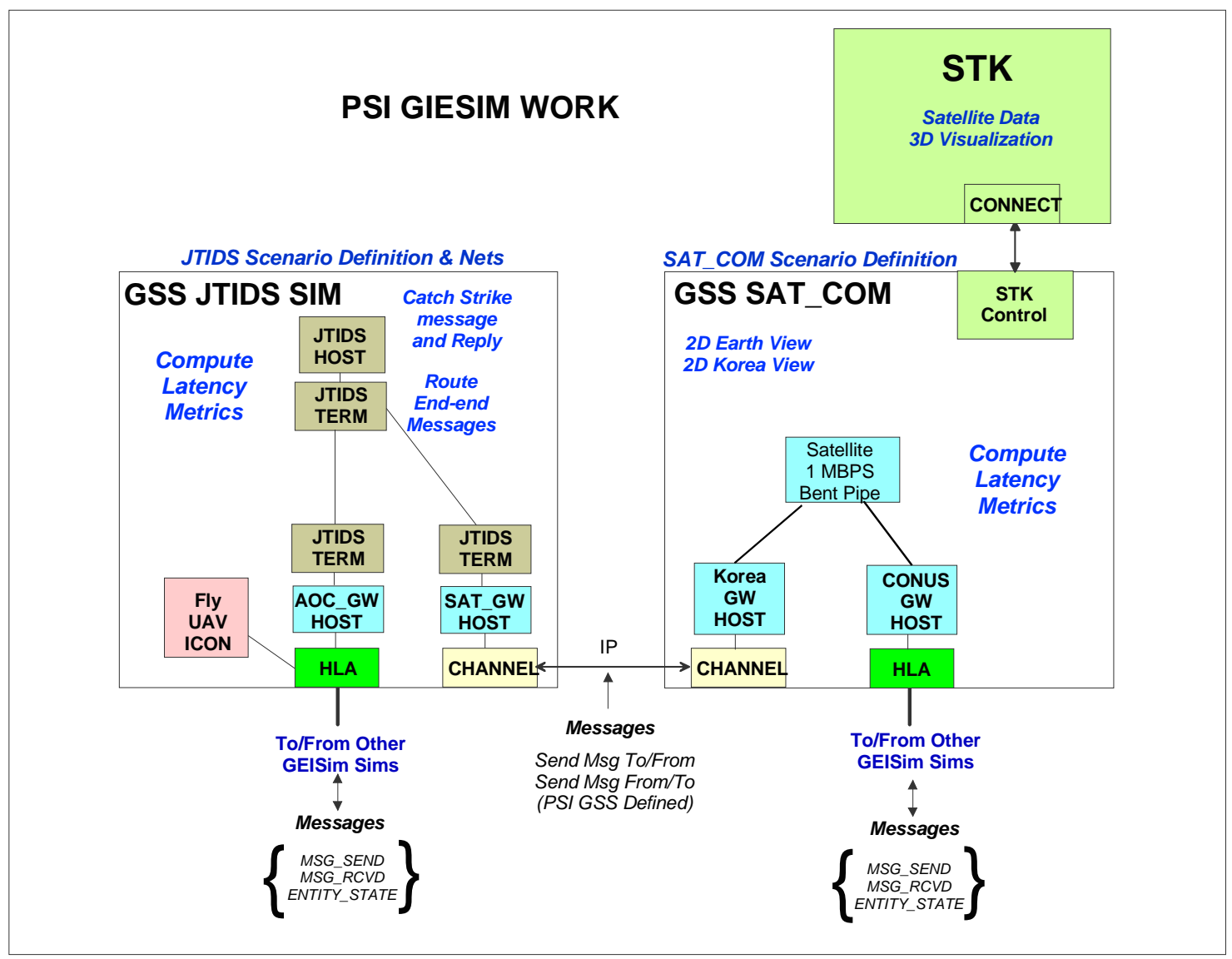

Figure 4 - PSI Work Accomplished for GIESim SAB Demo

The PSI JTIDS Simulation was modified to include two gateways to route messages through the JTIDS network. Specific Operational Nets were defined for this purpose as discussed in a later section. The AOC Gateway (AOC_GW) routes messages received from HLA through JTIDS to either an F15 Strike platform or to the Korean Satellite Gateway (SAT_GW) for sending to the CONUS. The JTIDS SAT_GW connects to the PSI SAT_COM simulation via a GSS IP Channel interface. Conversely, messages received from SAT_COM via the IP Channel interface are routed by the SAT_GW through JTIDS for delivery at the AOC_GW. The AOC_GW then publishes an HLA interaction for reception by another GIESim Simulation player. In addition, the AOC_GW can receive a reply message over JTIDS from a Strike Platform for publishing over HLA.

The PSI SAT_COM Simulation was modified to include a control interface for use with STK, and gateways to buffer and route messages from the IP Channels and HLA interfaces over the satellite network. Furthermore, PSI SAT_COM was modified to show a 2D representation of both the whole earth and Korea.

Each PSI simulation computes it's own contribution to message latency and adds this to the accumulating message latency prior to sending over the network interface. 
In addition to developments completed within JTIDS, SAT_COM, and STK, PSI set-up a hubbased, network architecture similar to that planned for the GIESim SAB Demo in order to debug and validate models and simulation performance prior to the dry-run in Rome. 


\subsection{HLA INTERFACE AND TEST_DRV DEVELOPMENT}

PSI developed a GSS implementation of the five HLA Interactions defined for the GIESim SAB demo. The GSS architecture for the HLA interface is shown in Figure 14 in Appendix A. GSS provides built-in support for HLA interactions making the architecture to support the GIESim HLA interactions rather simple. For each HLA interaction, we define a resource with the same name as the interaction - this automatically subscribes GSS to this particular interaction name. We also define an HLA event handler that handles HLA subscribe events by calling the appropriate process for the received interaction. To publish an interaction, we load data into the resource and PUBLISH it. GSS automatically generates the “.fed” FOM file.

As a means to test and verify the HLA implementation, PSI developed an HLA Test Driver to exercise the publishing and subsequent subscribing of the HLA interactions. Figure 15 in the Appendix shows this GSS architecture. This test driver makes heavy use of GUI panels to display published and subscribed data. The use of panels in GSS is very easy, and makes understanding data easy compared to simple print statements. The architecture of the complete TEST_DRV simulation that combines our HLA implementation with the HLA Test Driver is shown in Figure 15 in the Appendix. TEST_DRV also contains developmental models for using the GSS Channel capabilities between JTIDS and SAT_COM. This functionality is not currently exercised in TEST_DRV. The HLA Test Driver uses a main control panel to display publish and subscribe statistics. This panel is shown in Figure 5.

This panel presents a count of all interactions published and received, and presents separate counts of published and received events for each of the five interactions. The Message Window shows HLA traffic activity and stamps each event as a "PUB" or "REC", its interaction name, and time of occurrence. Published events are tagged with an ' $A$ ' for an automatically generated event or with an ' $M$ ' for a manually generated publish event.

As shown in Figure 5, this panel provides buttons to manually publish individual interactions and check boxes to cause each interaction to be generated automatically. The check boxes labeled "DISP" enables the display of separate panels that show data for interactions as they are published and received. Examples of the panels for the ENTITY_STATE interactions are shown in Figure 6. The left side of Figure 6 shows a manual publish data panel for one instance of TEST_DRV with ID 56, and right side of Figure 6 shows a receive data panel for an instance of TEST_DRV with ID 337. 


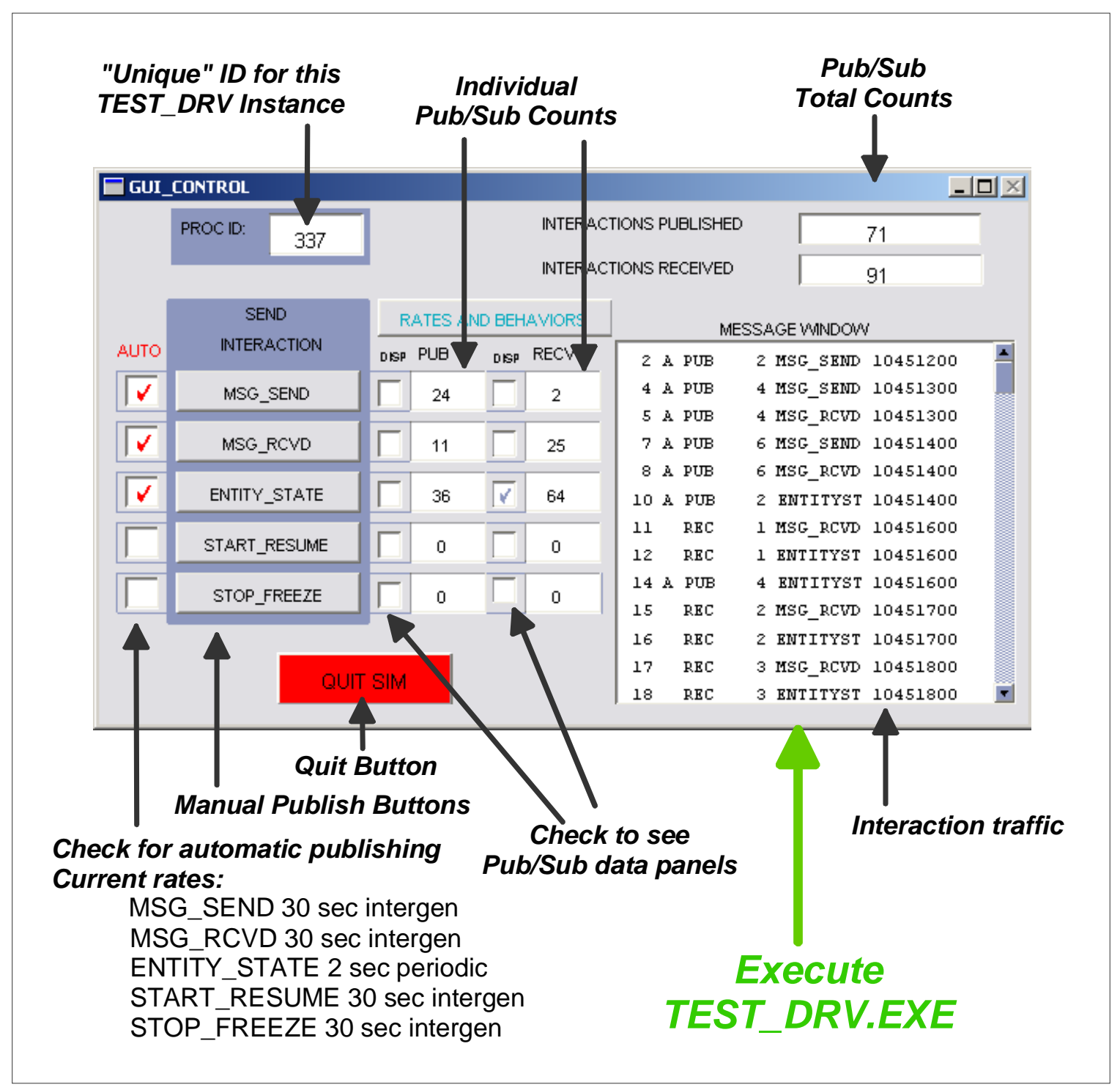

Figure 5 - Main GUI of PSI's HLA Test Driver - TEST_DRV

The TEST_DRV tool is designed to be executed multiple times so that one instance can publish an interaction and another can "catch" it as a subscribed event. Each data panel is labeled with the ID for the TEST_DRV instance it is associated with.

TEST_DRV was used for interoperability testing that took place with other GIESim team-member simulations. The components of TEST_DRV were incorporated into the PSI JTIDS and SAT_COM simulations to handle pub/sub of HLA interactions with the other simulations in the GIESim SAB Demo. The GUI panel capabilities were retained in JTIDS and SAT_COM to facilitate testing and data verification whenever needed. During the SAB demo, these panels will be hidden unless there is value to showing the HLA exchange of messages. 


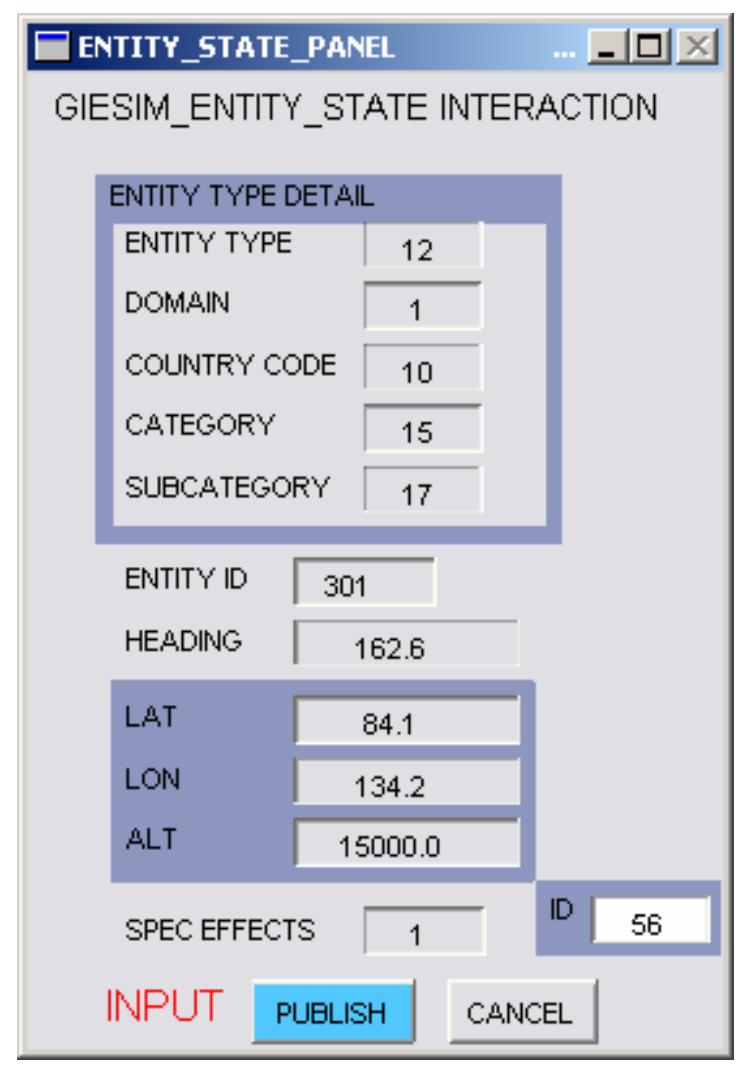

Publish Data Panel For TEST_DRV ID 56

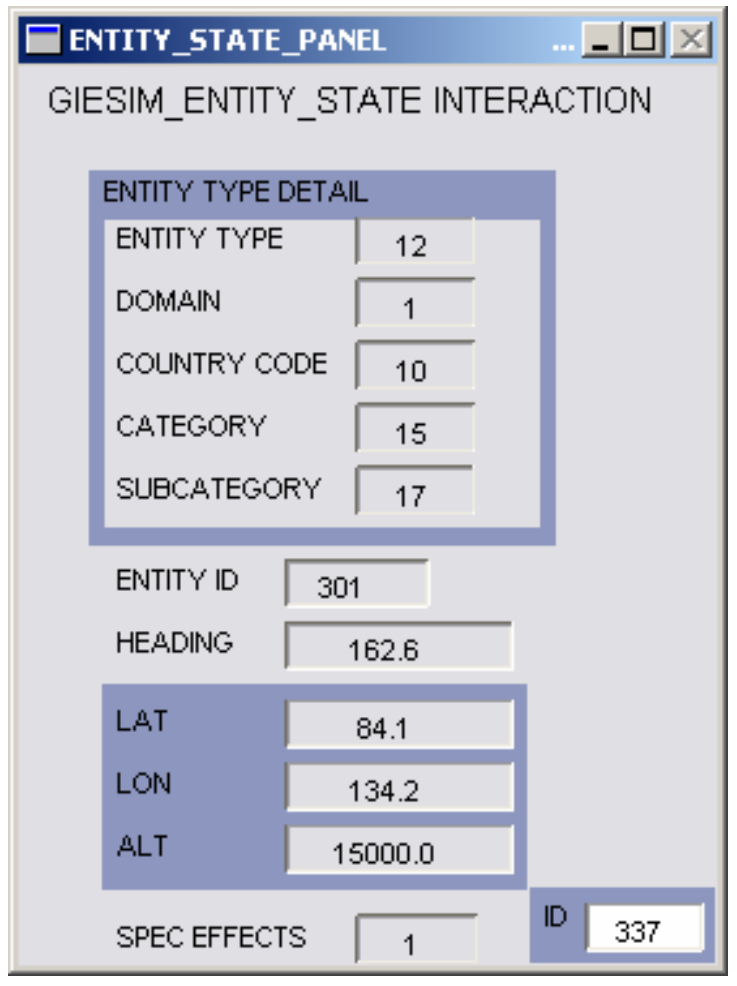

Receive Data Panel For TEST_DRV ID 337

Figure 6 - GSS Panels for Pub/Sub of HLA Interactions 


\subsection{JTIDS SIMULATION DEVELOPMENTS FOR GIESIM}

PSI completed the JTIDS model development and integration required for the GIESim SAB Demo. Figure 7 shows a screen shot of the modified JTIDS simulation. The following work items were completed on the JTIDS simulation for GIESim:

1. The Korean package scenario was modified to include an AOC Gateway (AOC_GW) and SAT Gateway (SAT_GW). Both are equipped with JTIDS radios. These platforms are represented as icons as shown in Figure 7. Each gateway has a receive and transmit buffer. Messages that exceed available buffer capacity are dropped. Messages are sent across the JTIDS network at the rate and in segments sized for the characteristics of the operational net being used. The gateways perform message segmentation and reassembly, and count messages sent, received, dropped (buffer full) or lost (in the JTIDS network). Associated with each gateway are panels that show buffer size, current fill level, and counts of sent, received, dropped, and lost messages. The panels are updated dynamically as conditions change. Figure 8 shows these panels in more detail. Note that each panel was captured at a different time. The background coloring of the panel is intended to reflect the source of the message traffic. Panels for messages originating from a ground source are yellow color. Panels for messages coming in over JTIDS are colored blue. The bright color scheme was chosen for impact and clarity.

2. Sets of Operational Nets were defined with the PSI JTIDS Operational Network Planning Tool for use in the SAB Demo. Table I lists these nets along with their characteristics.

a. Nets 104 and 107 are high speed nets for fast transmission of image data, and have a maximum throughput of 19.2 Kbps.

b. Nets 103 and 106 are very slow nets and were intended to demonstrate image transmission latencies that were much longer than the performance requirements. In dryrun testing, the high speed nets are found to be better suited to the SAB Demo, so use of the low speed nets was dropped.

c. Net 105 is intended to convey a message to an F15 strike platform from the AOC, and Net 102 is intended to convey a response from the strike platform.

Table 1- JTIDS Operational Nets Defined for GIESim SAB Demo

\begin{tabular}{|c|c|c|c|c|c|c|c|c|c|c|c|}
\hline \multirow[b]{2}{*}{ Net Name } & \multirow[b]{2}{*}{ Net ID } & \multirow[b]{2}{*}{ Src } & \multicolumn{4}{|c|}{ GIESim Demo - JTIDS Nets } & \multirow[b]{2}{*}{ Words/Msg } & \multirow[b]{2}{*}{ Msg per-> } & \multirow[b]{2}{*}{$\mathrm{Sec}$} & \multirow[b]{2}{*}{$\begin{array}{c}\text { Resp } \\
\text { rate }\end{array}$} & \multirow[b]{2}{*}{ Relay? } \\
\hline & & & Dest & Type & Packing & Encoded & & & & & \\
\hline Slow Image Net & 103 & AOC_GW & SAT_GW & Free Txt & P2 & No & 6 & 1 & 10 & 10 & Yes \\
\hline Slow Image Net & 106 & SAT_GW & AOC_GW & Free Txt & P2 & No & 6 & 1 & 10 & 10 & Yes \\
\hline Fast Image Net & 104 & AOC_GW & SAT_GW & Free Txt & P4 & No & 12 & 21 & 1 & 2 & Yes \\
\hline Fast Image Net & 107 & SAT_GW & AOC_GW & Free Txt & P4 & No & 12 & 21 & 1 & 2 & Yes \\
\hline Weapons Coord & 105 & AOC_GW & F15 & FWF & P4 & Yes & 3 & 1 & 12 & 2 & Yes \\
\hline Weapons Coord & 102 & F15 & AOC_GW & FWF & P4 & Yes & 3 & 1 & 12 & 2 & Yes \\
\hline
\end{tabular}




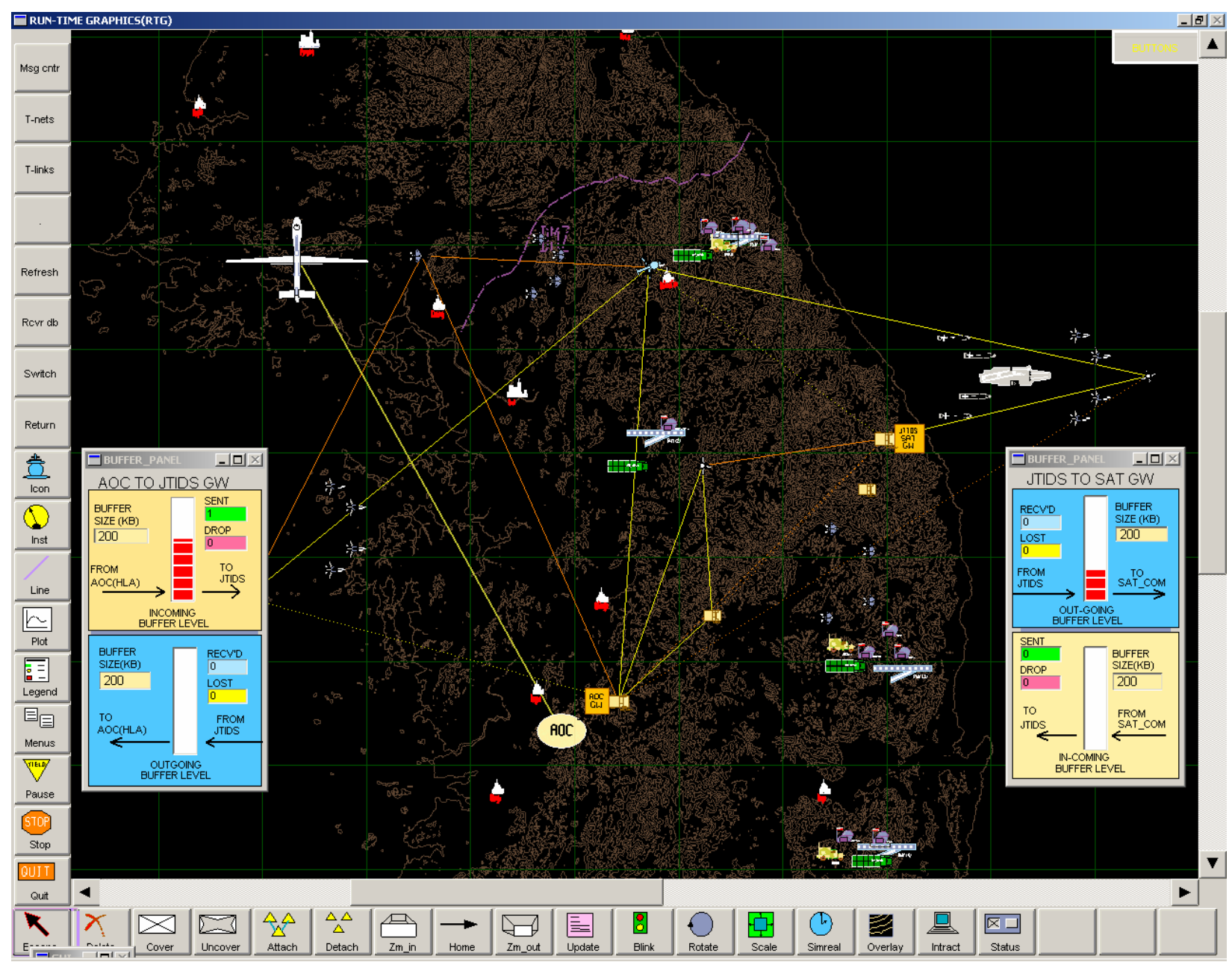

Figure 7 - JTIDS Simulation with GIESim Demo Modifications
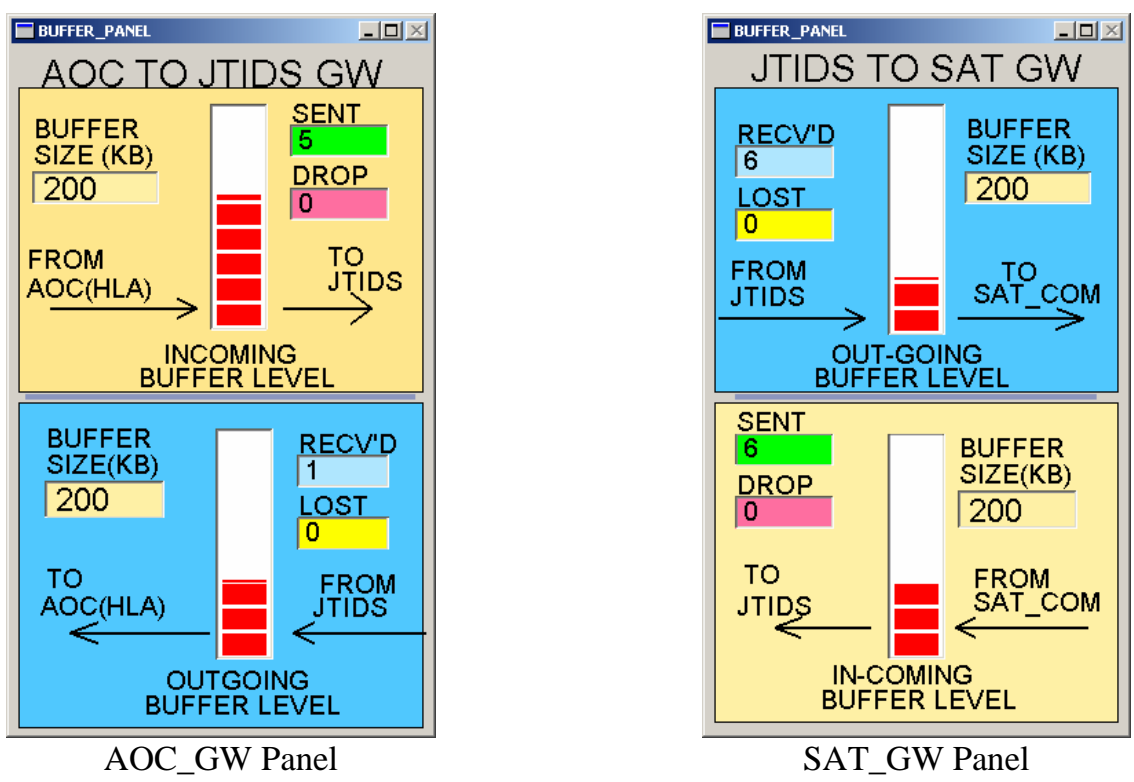


\section{Figure 8 - JTIDS Gateway Panels}

3. The new Operational Nets for GIESim were combined with existing operational nets for the Korean scenario and were then run through the PSI Time Slot Assignment tool, which automatically determined time slot assignments for all the nets. The resulting time slot assignments were then used in the JTIDS Simulation. In Figure 7, the nets for the AOC_GW, SAT_GW, and Strike Platform are shown as orange (direct path) and yellow (relayed path).

4. The JTIDS Simulation is set-up as an IP Server for a pair of IP Channels that connect it to the SAT_COM simulation. Two channels were used to permit full duplex message passing between the simulations, this allows messages to pass simultaneously in both directions.

A model was added to the JTIDS simulation to "fly" a UAV Predator icon as directed by HLA Entity State interactions received (i.e., subscribed to) from another simulation. The UAV icon can be seen in Figure 7. In addition, an icon for the "AOC" was created and added to the simulation to illustrate the AOC in Korea, and a dynamically updated line connects the AOC icon with the UAV icon as it moves.

As a result of the August interoperability dry-run at AFRL Rome, it was concluded that dynamic setting of the JTIDS Gateway buffer sizes would be desirable, as this capability would improve the SAB Demo. Consequently, a GSS function button in the JTIDS simulation was programmed to invoke a Panel for changing the size of the ingoing and outgoing buffers on both the AOC and SAT gateways. Figure 9 shows this new Panel along with the new function button and the AOC Panel before and after buffer sizes were changed.

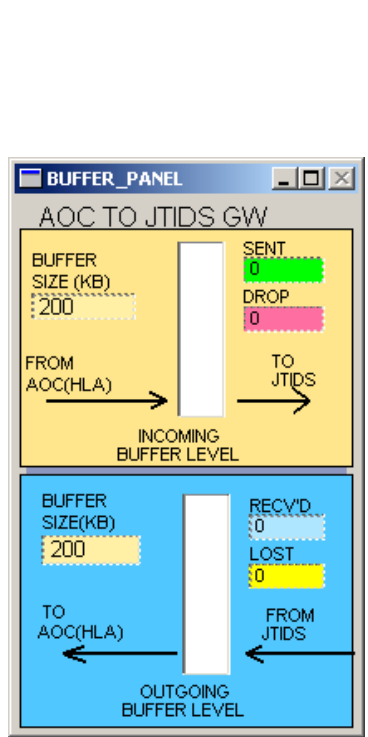

Initial Buffer Sizes

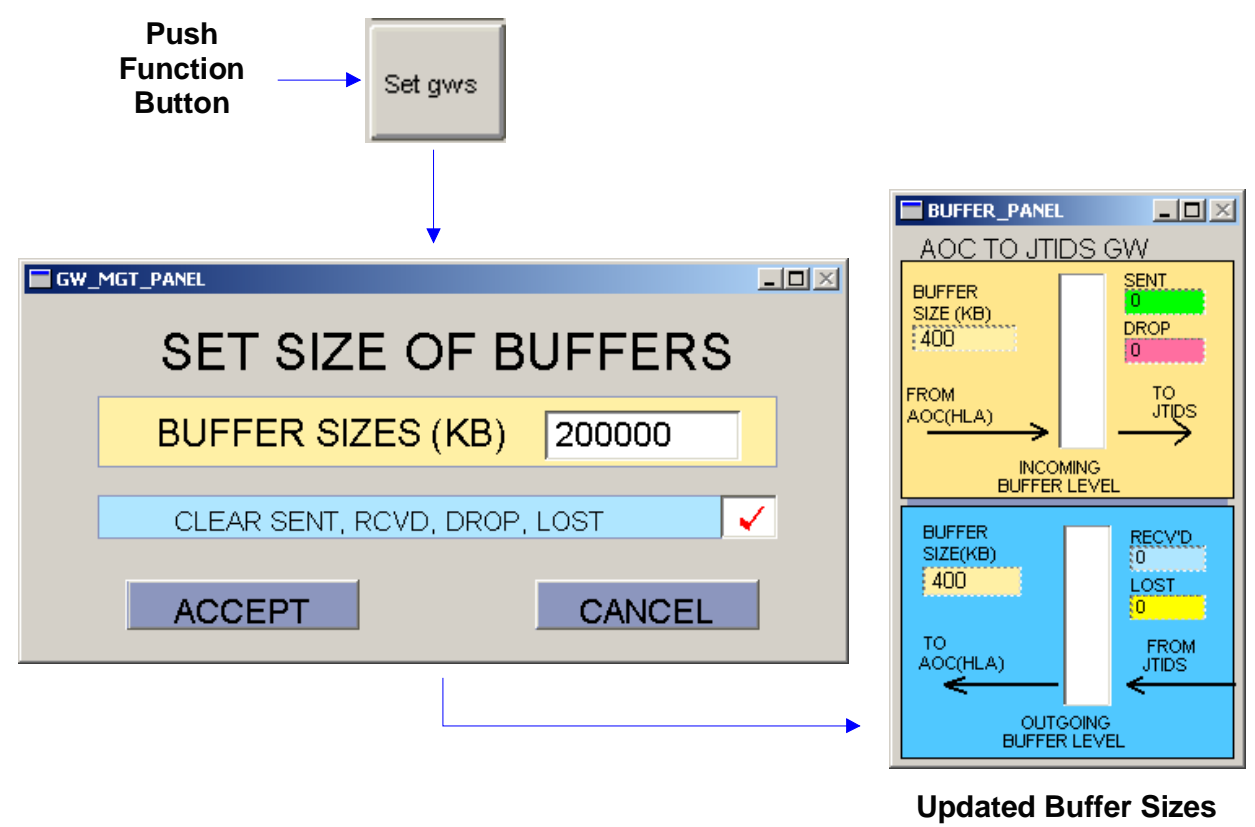

Figure 9 - JTIDS Gateway Buffer Size Panel 


\subsection{SAT_COM SIMULATION DEVELOPMENTS FOR GIESIM}

Several modifications were made to the PSI SAT_COM Simulation in support of the GIESim SAB Demo. The most notable modification was the development of an interface to STK to obtain satellite orbit and connectivity data. The following list presents the work accomplished on PSI SAT_COM for the GIESim demo:

1. PSI completed the interface between SAT_COM and STK using the IP CONNECT interface on STK. This work involved the addition of STK APIs into SAT_COM, understanding and loading the appropriate data structures to send to STK, and unpacking of data structures received from STK. PSI set-up the STK server to show several views (2D and 3D) of the satellite constellations with respect to the earth once the SAT_COM client connected to it. SAT_COM does the following with respect to STK:

a. Initializes the STK CONNECT link and opens the STK Viewer on a separate PC running a licensed copy of STK provided by AGI.

b. Uploads data on the location of ground stations and initial satellite positions.

c. Downloads tables of satellite position and connectivity data. This essentially compresses a four day scenario into two hours, which makes for a more interesting (dynamic) display.

d. Uses downloaded information to generate it's own satellite connectivity lines and positioning of satellite icons.

2. An HLA interface was added to SAT_COM to subscribe to and publish GIESIM_MSG_SEND interactions. This interface is for communication with other simulations in the GIESim SAB Demo. This interface can also monitor and generate the other GIESim HLA interactions if needed.

3. Two GSS Channel Client interfaces were added for bi-directional communication of messages to and from the PSI JTIDS Simulation.

4. A Korean gateway was added to SAT_COM that will handle messages from the PSI JTIDS Simulation via the Channel interface and send them on the satellite "uplink" in Korea. Messages received on the Korean "downlink" are sent to JTIDS through the Channel interface. This gateway has panels that show buffer fill levels that are updated dynamically.

5. A gateway located in CONUS was added to SAT_COM to handle messages from/to the external simulations connected by HLA. This gateway receives message via HLA and sends them on the satellite "uplink" to Korea, and gets messages from the satellite "downlink" in CONUS then publishes them as HLA interactions. This gateway also has panel that show buffer fill levels that are updated dynamically.

6. SAT_COM was modified to show two different background overlays. One shows a 2D view of the whole earth, and the other view shows the terrain of Korea. Views are selected through a button in the graphics window. Both views support dynamic updates of satellite connectivity lines between satellites and to ground stations. Figure 10 and Figure 11 are screen shots from SAT_COM of the world and terrain views respectively. 


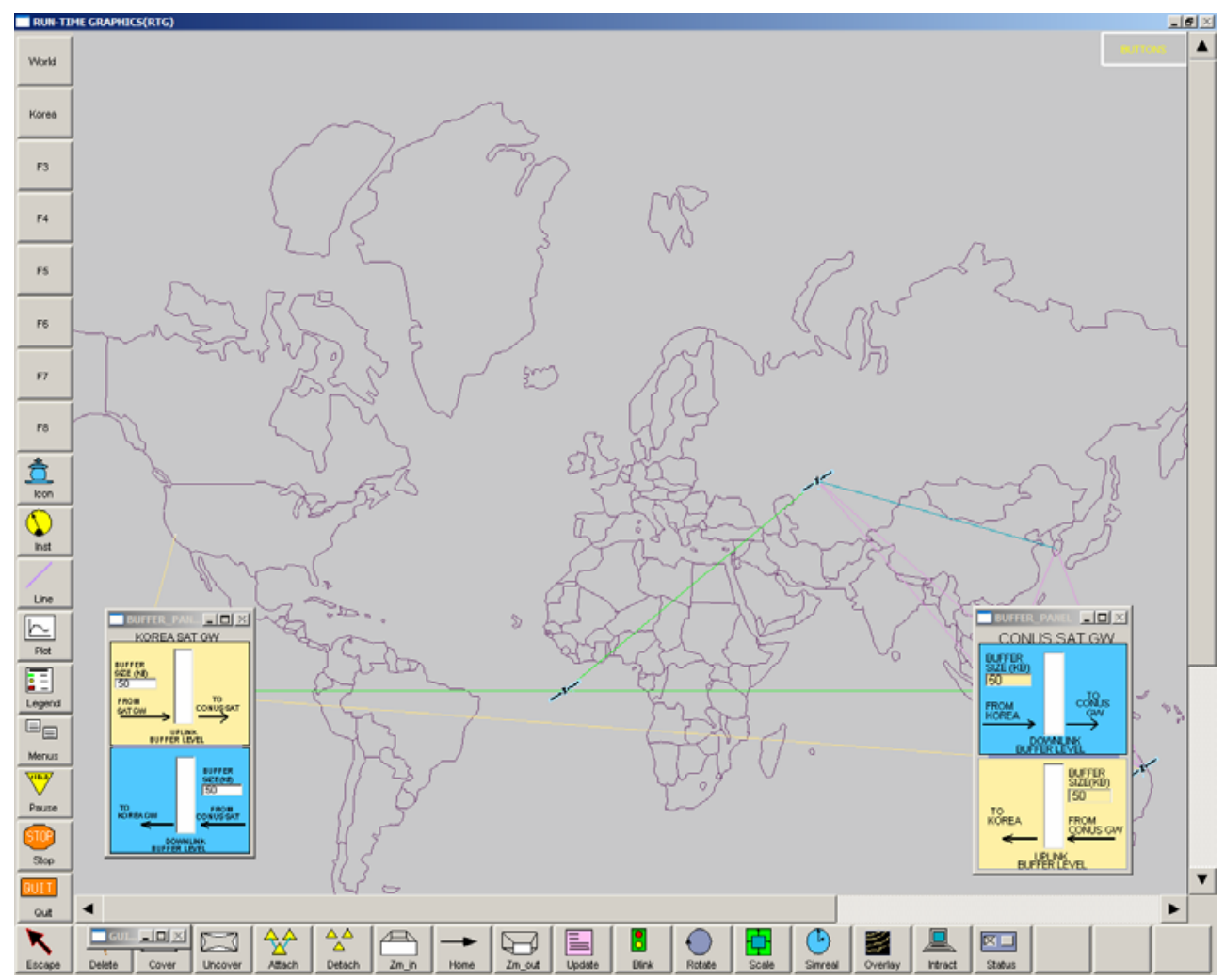

Figure 10 - SAT_COM World View

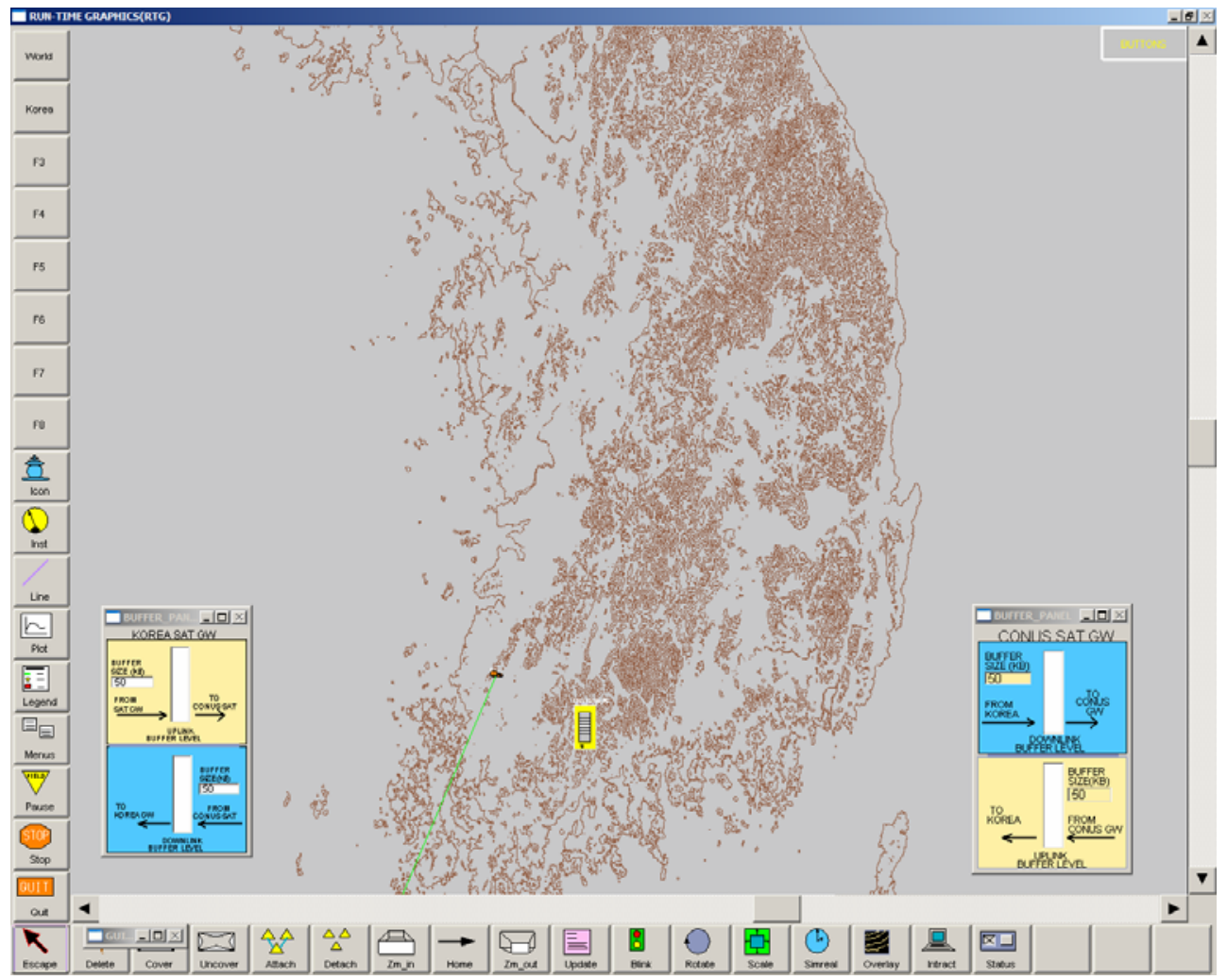




\section{Figure 11 - SAT_COM Korean Terrain View}

\subsubsection{STK Interface and Data for GIESim}

As mentioned in a prior report, PSI negotiated with AGI to obtain licenses for STK 5.0 for use in the GIESim SAB Demo. STK 5.0 provides API and interfacing descriptions for their IP-based CONNECT interface. PSI studied this material and built an interface to the CONNECT interface into SAT_COM. PSI completed the following work on STK for the GIESim SAB Demo project:

1. Obtained licenses and installed STK on two PSI machines - one of which is dedicated to the GIESim project and demo.

2. Tested the STK CONNECT interface and set-up several views in the STK Server/Viewer for the GIESim Demo.

3. Uploaded data on ground station locations and satellite data to STK.

4. Downloaded satellite orbital and connectivity data from STK.

5. Tested with SAT_COM and in conjunction with JTIDS and a Test Driver simulation.

Figure 12 shows the STK screen showing views of the satellite orbits defined.

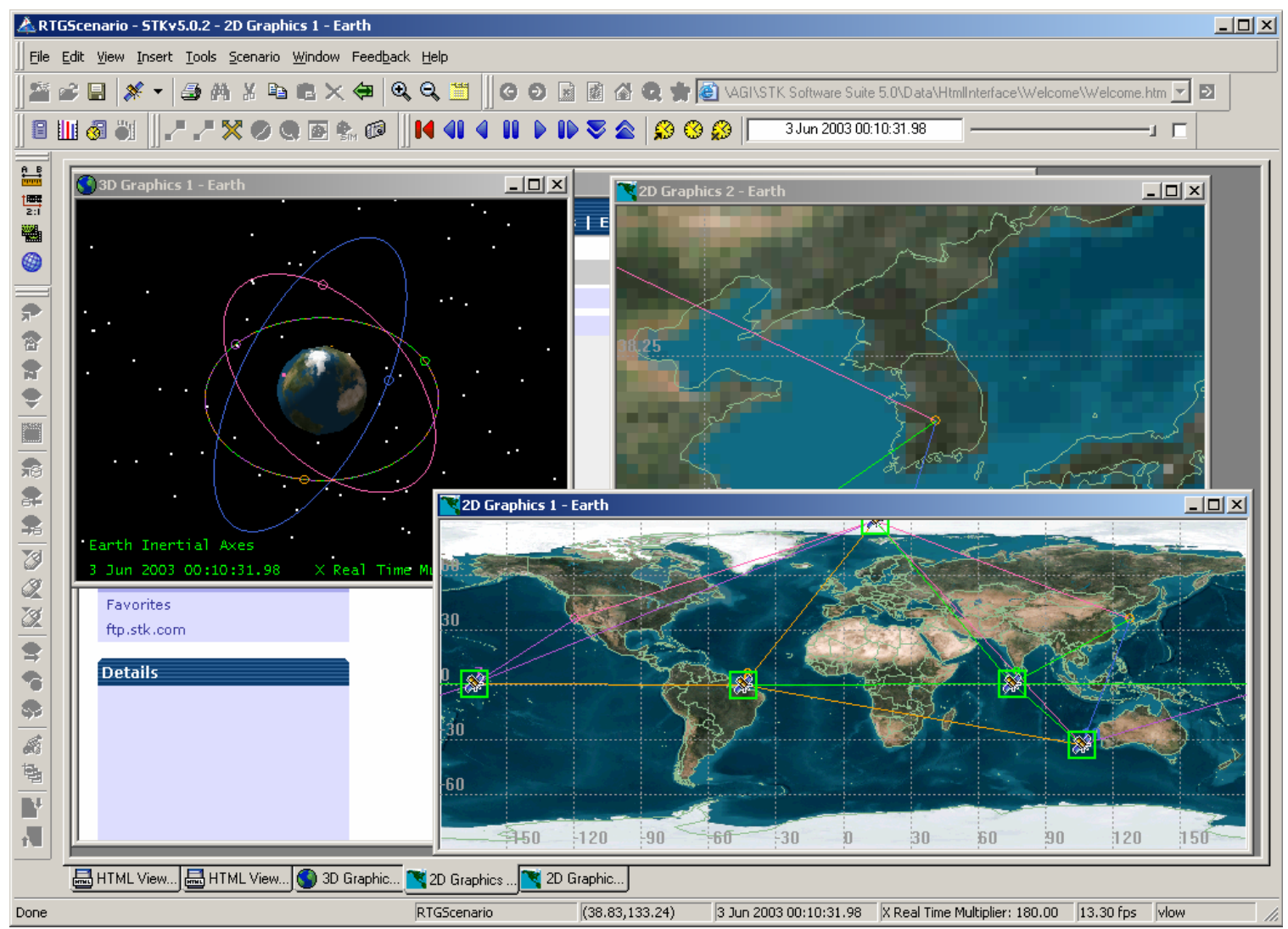

Figure 12 - STK Screen Shot for GIESim 


\subsection{SIMULATION TESTING BY PSI}

\subsubsection{In-House Testing}

In order to test the various simulation components built by PSI for GIESim, PSI assembled a test configuration very similar to that planned for the SAB Demo. This configuration is shown in Figure 13. Four PC's were used, with each dedicated to one function or simulation. Each PC was configured with an appropriate IP address, and the HLA RID files and Server IP addresses were configured accordingly. One PC (PC2) ran the RTI and served as a source for generating HLA interactions for testing. PC2 also subscribed to all GIESim interactions and, therefore, monitored and verified correct publishing of HLA interactions by the respective simulations.

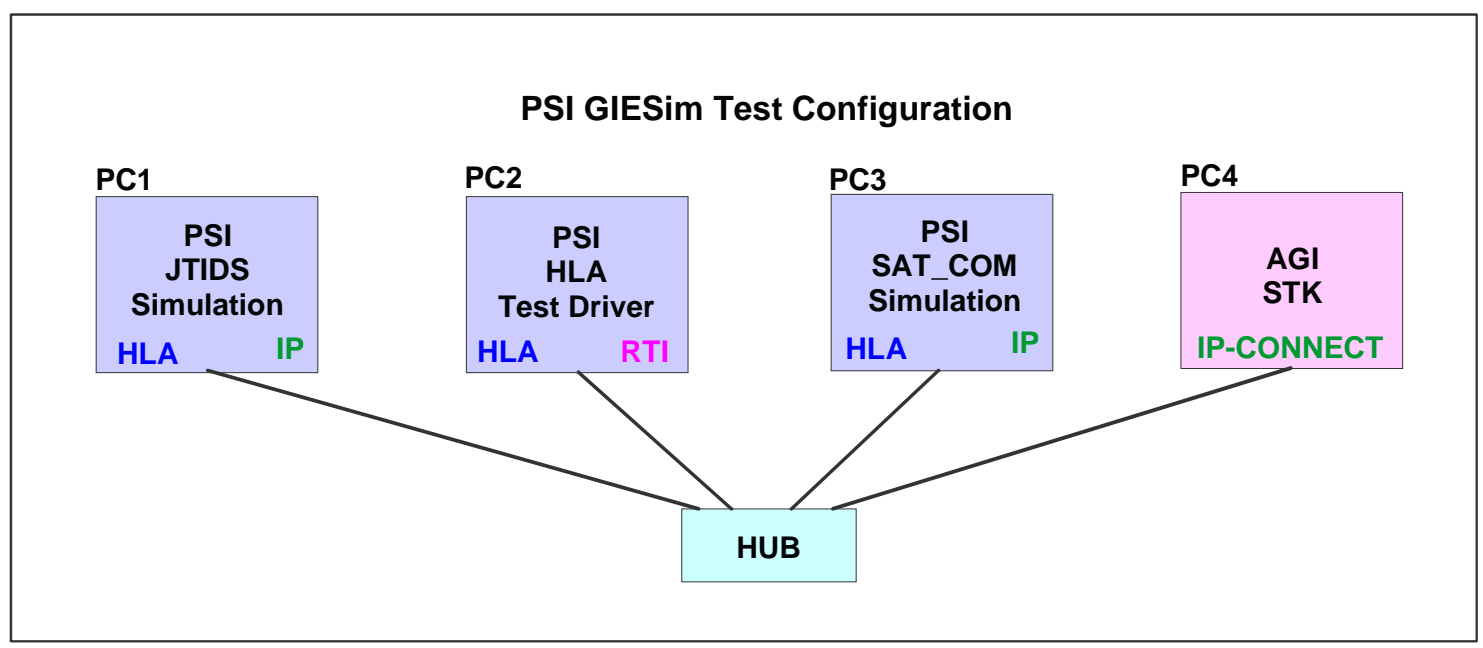

Figure 13 - PSI In-House Test Configuration for GIESim Work

Testing proceeded by using PC2 to generate HLA interactions first intended for JTIDS. The incoming message arrival and subsequent transmission through JTIDS and arrival at the SAT_GW were tracked. The resulting message was then tracked as it arrived at SAT_COM through the GSS Channel Resource and "flowed" through SAT_COM to the CONUS Gateway that published the message as an HLA interaction. PC2 was then used to test in the opposite direction by publishing an HLA interaction intended for the CONUS Gateway in SAT_COM. Message flow was followed through SAT_COM, into the SAT_GW in JTIDS, through the JTIDS network and into the AOC_GW in the JTIDS Simulation where it was published as an HLA interaction. Latencies were checked along with HLA interaction ID fields.

PC2 also published HLA interactions for the F15 Strike Platform in JTIDS through the AOC_GW and verified that it replied to the AOC_GW by observing a GIESIM_MSG_RCVD interaction published by the AOC_GW.

PC2 was also used to generate multiple messages to verify correct behavior of the gateways and transmission through JTIDS and SAT_COM. PC2 was also used to generate extraneous HLA interactions to verify that JTIDS and SAT_COM appropriately ignored them.

The test configuration was also used to verify correct "flying" of the Predator UAV in response to received GIESIM_ENTITY_STATE interactions. The Test Driver was modified to generate GIESIM_ENTITY_STATE interactions with LAT LON coordinates associated with a circular flight path centered on Korea. 
Several refinements were made to a few internal components and were then verified to be operating correctly. In addition to the HLA Test Driver on PC2 having the ability to subscribe or publish HLA interactions, both JTIDS and SAT_COM have the same capability since they employ the same HLA interface developed by PSI for use in the GIESim SAB Demo. This allows tracking and publishing of HLA messages from each simulation to verify message arrival (and publishing) as needed. This feature of the modified JTIDS and SAT_COM simulations proved useful during the dry-run testing that took place in Rome in late August.

\subsubsection{Internet HLA Interoperability Tests}

On July 29, 2003 an HLA interoperability test took place between a simulation running at PSI in NJ and a simulation running at SAIC in Ohio. SAIC ran the RTI in Ohio. After a couple of very minor glitches, all five interactions were successfully published and subscribed, and over 1000 interactions were successfully exchanged. All fields and sub-fields of the five HLA interactions were validated in the exchange. PSI sent a copy of it's HLA Test Driver (TEST_DRV) to SAIC.

\subsubsection{Dry Run Interoperability Testing at AFRL Rome}

PSI took part in a successful dry-run of the multi-simulation demonstration at AFRL Rome on August $26^{\text {th }}$ and $27^{\text {th }}$. Only minor tuning of the JTIDS and SAT_COM simulations was needed. When the demo scenario was run we found that the low speed nets originally planned to demonstrate "failure to meet performance requirements" were really not needed. Instead, Dr. Debany felt that the ability to show dropped messages as a result of a gateway buffer being too small made for a more effective demonstration. Subsequently, the JTIDS simulation was modified to support dynamic, real-time user settable gateway buffer sizes. Also, the gateway GUIs were modified to show dropped (buffer full) and lost (JTIDS dropped) messages in addition to the number of messages successfully sent and received.

At the time of writing this Final Report, another dry-run of the full DMSD (including JBISim) is scheduled for Oct 14-15 at AFRL Rome. This should be the final "dress rehearsal" for the SAB Review now scheduled for mid November. 


\section{LESSONS LEARNED}

The DTIG Multi-Simulation Demonstration (DMSD) was built for the SAB Review as a proof-ofconcept or first demonstration of the GIESim concept of a modeling and simulation framework for satisfying the needs for complex communications and information system simulations. In addition, the process of defining, designing, building, testing, integrating and running the DMSD provided both validations, challenges and insights into the concepts and Framework of GIESim. The GIESim leadership team was interested in lessons learned from the creation of the DMSD. Lessons learned as stated in the list below are from the perspective of PSI gathered from working with the GIESim team and from developing additional models for our existing simulations. These "lessons" have been shared with the GIESim team, and for the most part reflect similar "findings" by the other team members. They have not been "endorsed" by the team, and are therefore solely the view point of PSI.

- It is an order of magnitude or more cheaper (in time and dollars) to assemble a multi-simulation communications capability by drawing from existing models and simulations.

- The use of existing models and simulations does not eliminate the need for domain or subject matter experts. There is still a need for experts to define and develop the target analytical system:

o Experts in the models and simulation environments and tools

$0 \quad$ Experts in the systems being modeled

0 Expertise in reducing system needs and requirements to the selection of appropriate models/simulations/environments and scenarios. (See below)

- Model requirements and multi-simulation architecture must be defined based on requirements and needs of the target analysis that is to be accomplished. Validity should be looked at as a quality process with each simulation/model component impacting quality in a potentially multiplicative way.

- Existing models and simulations may (and probably will) need tailoring to meet the needs of the each experiment. Thus, a simulation environment that supports ease of use and understandability is essential. Good architectural designs are also needed. Some points to consider include:

o Definition and development of IP Interfaces, and HLA interfaces with associated Interactions, etc., with of IP Addresses and required simulation and entity IDs.

o Definition and development of metrics and capabilities to collect them, e.g., latency. These metrics may require new models or tailoring of existing model to gather and report to the multi-simulation environment.

o Definition and development of means to interact with a simulation to introduce controlled flaws, outages, etc. to explore overall impacts on the communications system being studied. Means might include real-time feeds, access to data files, user interaction with the running simulation, and multiple simulation runs.

o Definition and development of appropriate visualization capabilities to support both testing and analytical experiments.

- Scenario development and equipment collections must be defined and potentially developed for the multi-simulation environment. This will require:

o A common definition of what is meant by "scenario".

o Import of terrain for area of interest.

o Flight paths and other movement paths for dynamic communications.

o Definition and implementation of network characteristics (connectivity, network capabilities, redundancies, etc.).

o Traffic generators and other means to stimulate the simulation environment in realistic ways.

o The GIESim multi-simulation environment may need to interface with external simulation systems and take scenario inputs from them.

o Many other variations and considerations are likely. 
- Testing to ensure interoperability and validity is required and must be scheduled into the program. A layered approach can help to minimize the number of variables and conditions being tested.

- Potential modifications of models and simulations may be needed as identified during testing and during analytical experiments, e.g., need to have the ability to change buffer sizes. Ease of use of the simulation environment is critical for this.

- Many terms and parameters need to be defined. Here are a number of examples:

o We may often talk about multiple fidelity models, but what does fidelity really mean and how should we quantify it. Furthermore, how do (or should) we translate customer needs into an assessment of model fidelity requirements? How should the "fidelity" of one model be factored into the fidelity of other (potentially) interacting models when addressing the requirements for a communications simulation solution.

o Model and simulation performance needs to be quantified. The GIESim approach to building complex communications simulations can certainly cut development time and build times. In some (perhaps many) cases, execution times may be equally or more important. To attain statistical validity over a range of operating conditions and situations, a GIESim simulation environment will need to be exercised over many variations of scenarios and parameters. Hence, model and simulation execution performance may become critical factors in selecting the tools for a particular simulation.

o Performance versus fidelity trade-offs are often discussed. In some cases high fidelity models may run faster than low fidelity models, and therefore satisfy both speed and fidelity requirements.

As GIESim evolves, these kinds of lessons learned and newer considerations must be taken into account, particularly since there is a desire to automate and stream-line the entire simulation "realization process". The next section expands on this view and provide suggestions for future work leading to the success of GIESim. 


\section{SUGGESTIONS FOR FUTURE WORK}

PSI feels strongly that GIESim has great potential to be "the" place to go to get answers to complex communications and information questions that require sophisticated simulations to answer. In FY04, the AFRL GIESim leadership is looking to extend and expand on the work done in FY03 on the creation of the GIESim Architectural Framework. The themes for FY04 are along the lines of "faster, cheaper, better". More specifically, one objective is to "further refine and evolve the GIESim architecture to enhance support for the investigation and analysis of communication topologies for GIE”.

When it comes to architecture definition, requirements analysis, scenario planning and simplification of scenario developments, metric definitions, performance, etc., PSI feels strongly that it is important to consider the needs of real potential customers and systems. Such a "context" is vital to setting realistic directions for GIESim and for maintaining a good focus.

In addition to expanding on the DMSD, here are some considerations for future FY04 work:

- $\quad$ Focus on the needs of DTIG and those of other tactical systems to provide a more concrete and grounded framework for directing the evolution of GIESim, otherwise work may tend to get too abstract.

- $\quad$ Re-evaluate of the purpose of GIESim as originally laid out by Dr. Debany, and reflect on how our goals may have changed. This will keep us somewhat customer focused and goal oriented.

- Create a list of GIESim attributes that would attract customers and reflect highly on GIESim within both the scientific and operational communities. Perhaps listing what we see as benefits of GIESim from the perspectives of potential customers and clients is on par with the importance of integrating COTS and GOTS simulation components. Why should a "customer" care about GIESim, and what is the cost/benefit relationship that will bring clients to GIESim? Here are some of the potential "selling points" for GIESim:

o GIESim is an enabler of communications simulation and modeling needs. Dr. Debany once said that some people think simulation is too expensive and doesn't work, whereas others think all needed simulations are already done. Reality, as we know, is somewhere in between. GIESim can be a valuable source of what is possible in the world of building communication simulation solutions - for a reasonable price and in a reasonable time frame.

o GIESim can be used to analyze, cost out, assemble, integrate and deliver communications modeling needs of tactical and operational systems (existing and planned), and of large Force-on-Force simulations faster, cheaper, easier and with higher fidelity than through any other means.

o The GIESim team and lab has demonstrated the successful ability to bring disparate simulations together to prove its approach. The team will continue to accumulate expertise and models to make simulation solutions of complex communications needs be realized faster and cheaper.

o GIESim is the place to go to understand the state of the art in M\&S for communications systems.

o GIESim has codified and will continue to codify and refine the practices, approaches, standards, etc. to build simulation solutions quickly.

- With the above said, specific "customer" needs and some large Force level simulations should be explored to understand what they need and how we might need to interface with them. Doing so should help us with all aspects of the FY04 work from requirements analysis to development. Some potential customer needs might include:

o Execution speed

o Fidelity (how do they define this) 
o Types of questions they want answered and the time frames in which they need the answer, and the form they want the answers in: numeric, charts, real-time graphics, etc.

o What models, scenarios, inputs, metrics, etc. are required to support their needs?

0 What are their interface requirements? (systems, databases, user, etc.)

- $\quad$ Based on the above the GIESim team can then ask:

o) How does our "architecture" and current assembly of models and simulations support these customer needs? What changes may be needed?

o) What capabilities (documents, automation's, etc.) are needed to facilitate these kinds of customer needs?

The reason that PSI feels that a customer oriented approach is valuable is that it provides a much better context to address the kinds of things that are being discussed for the FY04 work program. For instance, with respect to looking for ways to "reduce the time required to implement scenarios....", this type of thrust is best addressed with respect to an investigation of actual needs, and opens questions about what is meant by a "scenario".

From the perspective of PSI, GIESim is ultimately (or should ultimately be) a provider of communications simulation solutions. We need to keep this in mind, otherwise our efforts could easily become an abstract software development in search "of" an application.

Another consideration for FY04 GIESim is testing. How do we determine that a complex arrangement of simulations is in fact working as intended? Testing and validation should be something that is considered going forward, particularly if GIESim gets some paying customers.

The GIESim concept and Architectural Framework are great and have the potential to fill the void in communications modeling and simulation needs that exist today, and that will become greater in the years ahead as the nature of war and military power changes and as the OPTEMPO continues to increase. Furthermore, the use of complex communications systems such as JTIDS is expected to increase, and the need for better, more timely, and cost effective communications modeling tools and Frameworks such as GIESim will grow. PSI is committed to the success of the GIESim effort and looks forward to working the GIESim team in FY04. 


\section{SUMMARY AND CONCLUSIONS}

PSI proactively participated in the definition and realization of the GIESim Lab multi-simulation demonstration for the SAB. PSI took part in team conference calls, and several face-to-face planning sessions in Rome. PSI made many contributions to the GIESim HLA Interactions that were defined for the GIESim HLA "backbone", to the scenario that was defined for the DMSD, and to the support material for the SAB Review. PSI designed, developed, and tested new interfaces and models for GIESim that were added to the GSS JTIDS and SAT_COM simulations. PSI took part in interoperability testing of the DMSD over the internet and at Rome on a number of occasions. PSI also built key GUI interface panels that effectively display activity of message traffic through the network. The JTIDS simulation in particular was the graphics visualization center piece for the DMSD.

Specific design, development and test work completed by PSI for the GIESim DMSD include:

- $\quad$ The addition of the following components to both JTIDS and SAT_COM:

o HLA and GSS Channel interfaces.

o Two gateway hosts in each simulation.

o Graphic interfaces in each to show message arrival and transmission.

- $\quad$ For the JTIDS Simulation:

o Defined Operational Nets to support communications between gateways for GIESim message traffic.

$0 \quad$ Added those nets to the nets already defined for the Korean scenario.

o Used the Time Slot Assignment tool to automatically generate time slots for all nets for use in the JTIDS Simulation.

0 Added unique icons for the AOC and SAT gateways.

o Added icons for an AOC and for a Predator UAV that is controlled via HLA.

- For SAT_COM:

o Developed an interface to control and use STK to obtain satellite orbit and connectivity data and to show 2D and 3D views.

o Added a 2D world view and view of the Korean terrain to SAT_COM for the GIESim SAB Demo.

- Conducted several tests of interoperability between the JTIDS, SAT_COM and STK using an isolated hub and an HLA test driver simulation.

- Took part in interoperability tests of our HLA interface over the internet, and at dry-runs of the DMSD in Rome.

Through monthly reports PSI documented progress and contributions to the GIESim project and to the DMSD. PSI contributed expertise in modeling and simulation gained through building solutions for many satisfied DoD customers to the evolution and success of GIESim. PSI contributed lessons learned in both documented and verbal forms, and was a co-creator of the SAB "story". PSI also participated in discussions and made contributions to the definition of a FY04 work program. PSI looks forward to continued participation with GIESim and to continued success with the program.

\section{APPENDIX A - SIMULATION ARCHITECTURES}

This appendix presents the simulation architectures of the various simulations employed by PSI for the GIESim DTIG Multi-Simulation Demonstration (DMSD). The architecture of each simulation is briefly described with specific additions and modifications that PSI needed to support the DMSD scenario highlighted.

One of the many unique and powerful characteristics of the General Simulation System (GSS) developed by PSI and used in the DMSD includes the ability to visualize software in a computer aided design (CAD) drawing. A simulation developer must define a simulation architecture via a GSS drawing 
before any software can be developed. PSI encourages development of architecture along physical lines, and GSS makes this easy to do. Another unique characteristic of GSS is the ability to define hierarchical models. This is analogous to the hierarchies often found in physical and natural systems. The architectures of the JTIDS simulation and of the SAT_COM simulation reflect the structure of JTIDS radios, and communications systems.

In GSS, model hierarchies can be nested as deeply as needed, and can be restructured easily as the simulation evolves. At the lowest levels of the model hierarchy are elementary models. Elementary models contain processes, e.g., rules that change state, and resources that contain data and state information. Processes are shown as rectangles, and resources are shown as rounded rectangles. Only those processes that are connected by lines to specific resources have access to data within those resources. It is this separation of processes from data that allows the software architecture to be drawn. Furthermore, once at the elementary model level, a user can immediately access process rules or data within resources with an editor by double-clicking on them. These features of GSS directly connect architecture to the underlying software! This is a powerful capability unique to GSS.

The ability to visualize simulation architectures dramatically improves comprehension of the simulation, which in turn dramatically improves productivity and also enables very effective software model reuse.

In the sections that follow, you will see that key model elements developed in support of DMSD appear in each of the PSI simulations. To support GIESim, PSI developed new models using a layered approach. These new models were then "imported" into the main JTIDS and SAT_COM simulations and tailored as needed. 


\section{A.1 GSS TEST_DRV SIMULATION}

The central design feature of the GIESim DMSD called for the use of HLA to interconnect the various simulation components from the different team members. This required the team to define a set of HLA interactions and associated simulation and entity ID numbers.

GSS has built-in support for HLA Interactions, which makes the use of HLA Interactions quite simple and easy. Figure 14 shows the HLA interface developed by PSI to handle the DMSD HLA Interactions. With GSS an HLA Event Handler is defined that fields published events. Events, in this case five HLA Interactions, are "subscribed" to by creating a resource of type "HLA" for each interaction and with the same name as the HLA Interaction. When an HLA Interaction is published by another simulation that matches one of the five HLA Interactions (resources), then the HLA Event Handler will call a process to handle that interaction. Also, the HLA resource can be "published”, which causes an HLA Interaction to be published.

Figure 15 shows the HLA Test Driver Model. This model was developed to test the PSI implementation of the GIESim HLA Interactions and contains the GUI Panels that were shown earlier in this Final Report. With GSS, constructing GUI interfaces is very easy and is a preferred means to present data and to take inputs from a user.

In order to exercise the HLA Interface and HLA Test Driver models, they were placed in a simulation named TEST_DRV. TEST_DRV was built so that several instances of the simulation could be running on a single PC, or on separate PCs. This allowed one TEST_DRV simulation to publish HLA Interactions for another TEST_DRV simulation to receive or "subscribe” to. This was an extremely effective means of testing PSI's implementation of the GIESim HLA Interactions, and was extremely useful in inoperability testing with the other GIESim simulations. We could very easily and quickly determine which of the HLA Interactions had been published and analyze message content. TEST_DRV can generate or publish HLA Interactions as needed to stimulate the other simulations during testing.

The hierarchical HLA Interface Model, consisting of the GIESIM_HLA_INTERFACE and the HLA_TEST_DRIVER models, were exported and then imported into the JTIDS and SAT_COM simulations. Hence these simulations were given the full capabilities of the TEST_DRV.

Also shown in Figure 15 are a number of other models. The UAV_FLYER model was designed to take HLA Entity State Interactions and "fly" an icon for a Predator UAV in the JTIDS simulation. The UAV_DRIVER was designed to test the UAV_FLYER. The other model shown is CHANNEL_INTERFACE. PSI had decided to exchange messages between the JTIDS and SAT_COM simulations using our IP Channel capability. This was done to demonstrate other means to interconnect simulations. These models were developed in TEST_DRV and exported to JTIDS and SAT_COM, and are not actively used in TEST_DRV. 


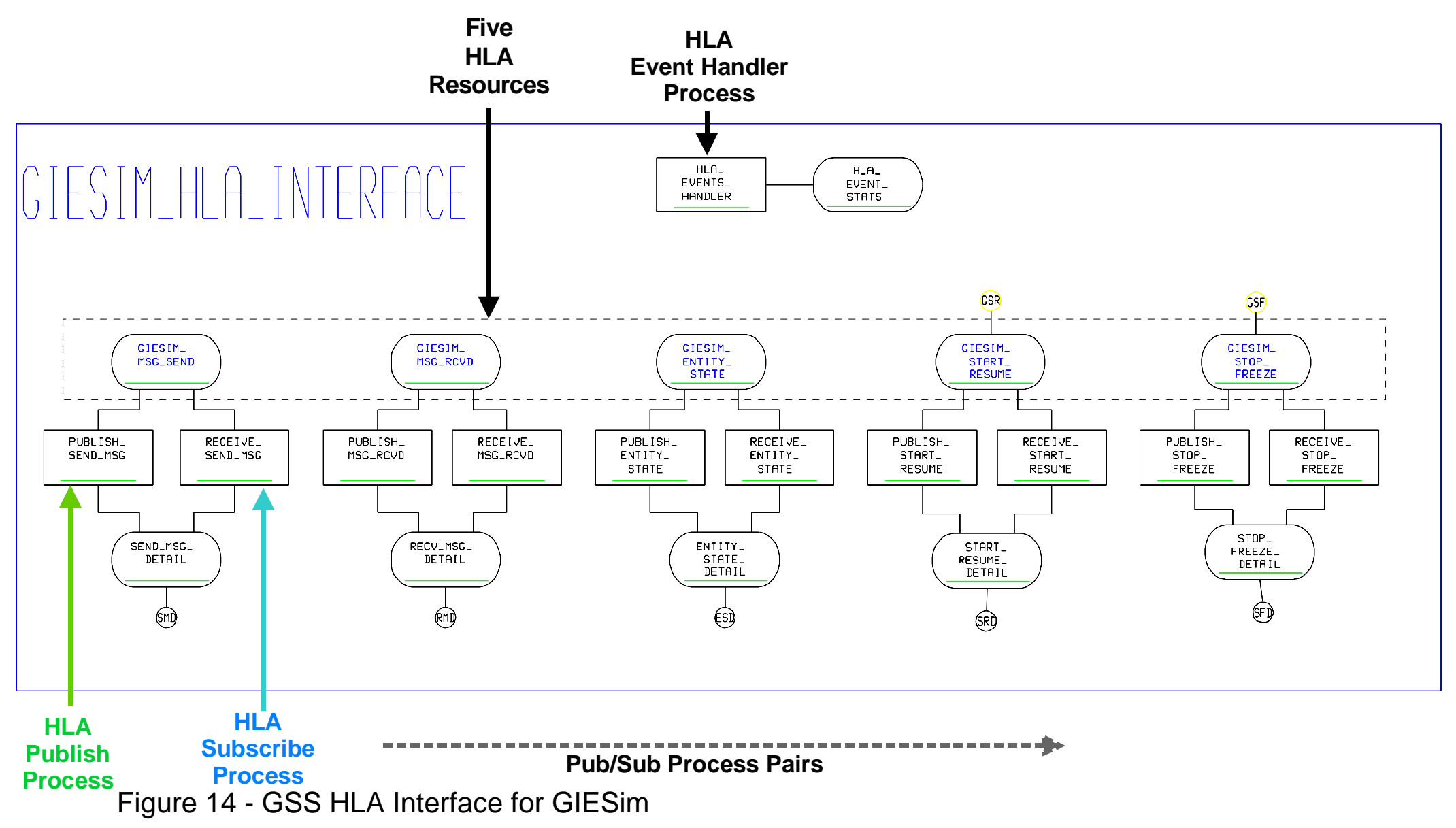


HLA

Pub/Sub Model

for GIESim HLA Interactions
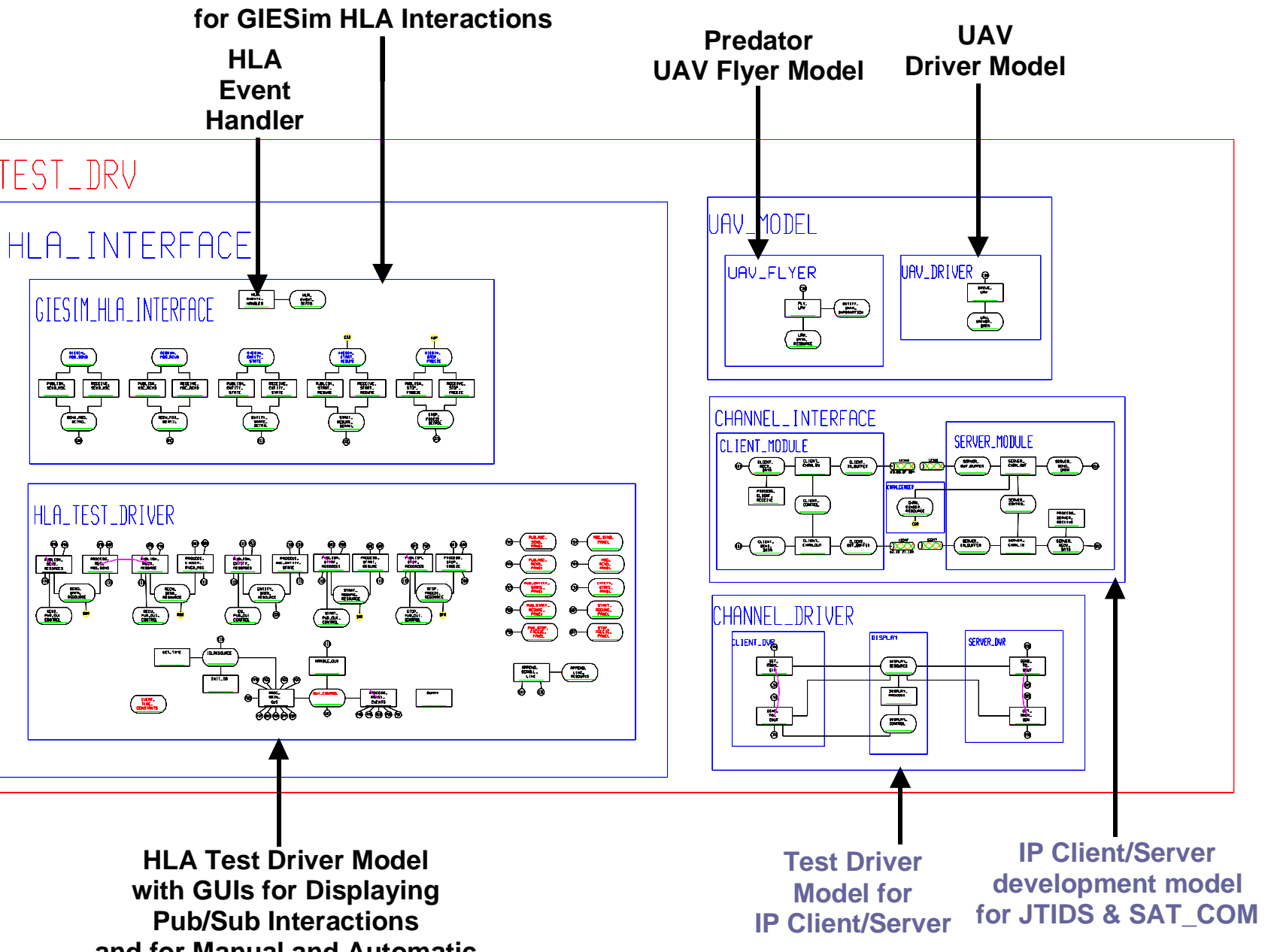

Generation for GIESim HLA Interactions

Figure 15 - PSI HLA Test Driver - TEST_DRV 


\section{A.2 GSS JTIDS SIMULATION}

The PSI created, GSS-based JTIDS simulation is an "industrial” or "operational” strength simulation of the complex JTIDS/Link-16 radio, and supports detailed simulation of message exchange over JTIDS Links and Operational Nets in complex equipment scenarios. JTIDS uses a highly accurate RF propagation model that accounts for the underlying terrain.

GSS JTIDS is the center piece of the GIESim DMSD. In addition to the detailed functionality it provides, it also offers a rich, interactive Run-Time Graphics (RTG) environment. RTG is a built-in capability of GSS. GSS JTIDS is the main visualization component within the GIESim DMSD. Figure 16 shows the architecture of the GSS JTIDS simulation, and indicates the portion of the complex model hierarchy that was added for GIESim, i.e., the GIESIM_INTERFACES model. In addition to the HLA Interface discussed in the preceding section of this Appendix, the GIESIM_INTERFACES model also contains:

- IP interfaces to the SAT_COM simulation (IP_SERVER_MODEL)

- A gateway to and from the AOC into the JTIDS network, and a gateway to and from the JTIDS network to the Satellite network (GIESIM_GATEWAY model)

- UAV_FLYER model to "fly" an icon of a Predator UAV over the Korean.

These models are shown in greater detail in Figure 17. JTIDS has two IP Servers that use the GSS IP Channel capability. One server is for messages into JTIDS from the SAT_COM simulation, and the other is for messages bound for SAT_COM from JTIDS.

The AOC and SAT_COM Gateways handle message segmentation and reassembly (SAR) that is needed to get a large UAV image message through the JTIDS network. The SAR function is sized by the characteristics of the JTIDS Operational Net being used. Each Gateway has a GUI Panel that shows inbound and out-bound message statistics (sent, dropped, lost, received) as well as buffer size and current buffer fill levels. These GUIs are updated dynamically. A separate GUI is provided to "resize” the gateways as needed to demonstrate the effect of the buffer being too small at first and then being increased to prevent messages being dropped. The Gateways are responsible for the calculation of message latency within JTIDS. Figure 18 shows the details of the AOC_GW Model and it's associated GUI Panel. The SAT_GW Model for the satellite gateway is virtually the same.

Also shown in Figure 17 is a small model that is responsible for connecting the GIESim message traffic into the simulated JTIDS network via the host interface in the GSS JTIDS simulation. Figure 19 shows the associated JTIDS Host Model changes made to support sending and receiving GIESim messages through the JTIDS simulation.

As can seen in these figures, a fair amount of model development work was required to support the requirements for the GIESim DMSD. GSS made this level of development quite fast and easy compared to other languages and approaches to simulation. As GIESim evolves some amount of new model development and model/simulation tailoring will likely be required to meet the visualization, scenario, and metrics requirements of systems being modeled. 
New Hierarchical Model added to JTIDS Simulation for GIESim

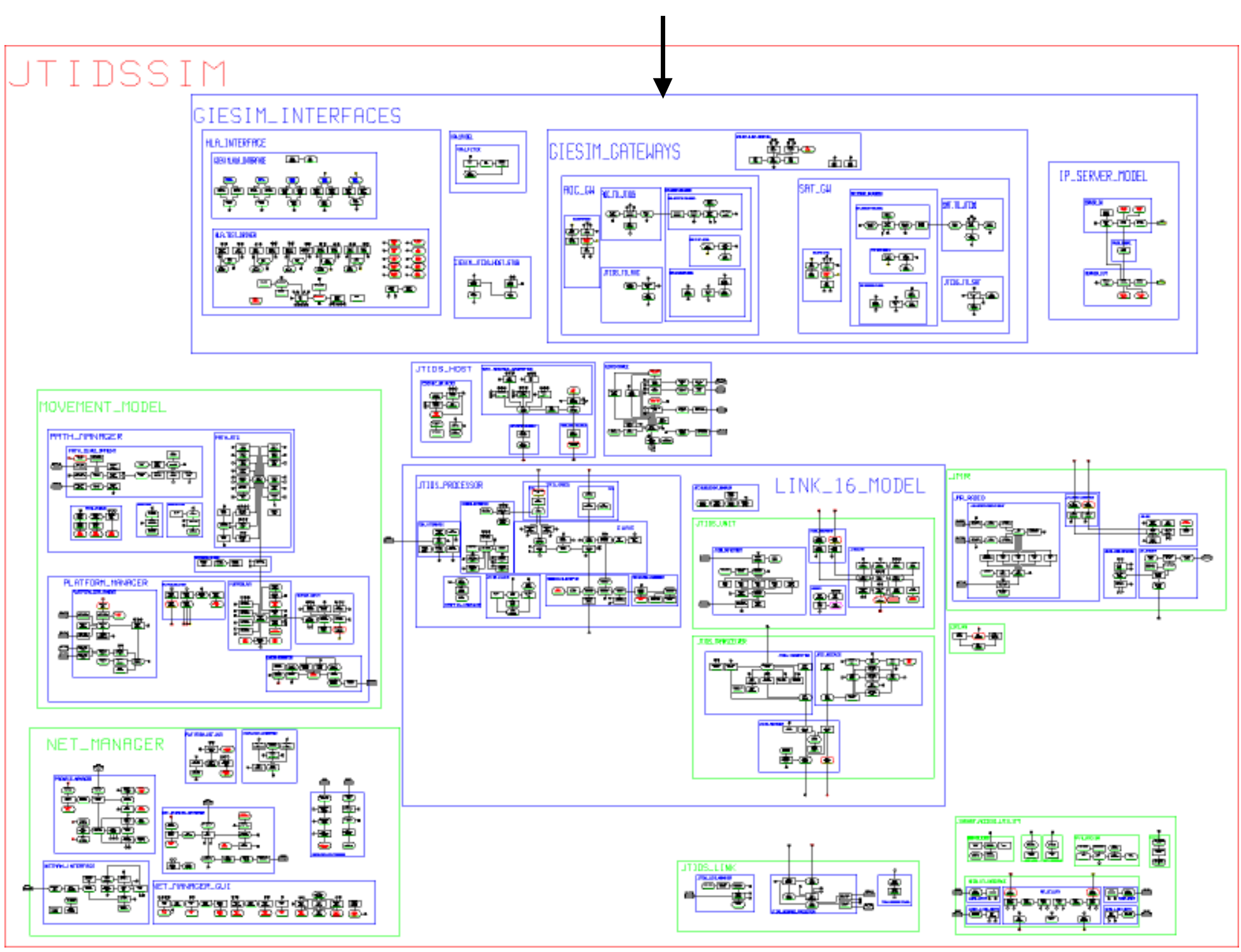

Figure 16 - GSS JTIDS Simulation Architecture 


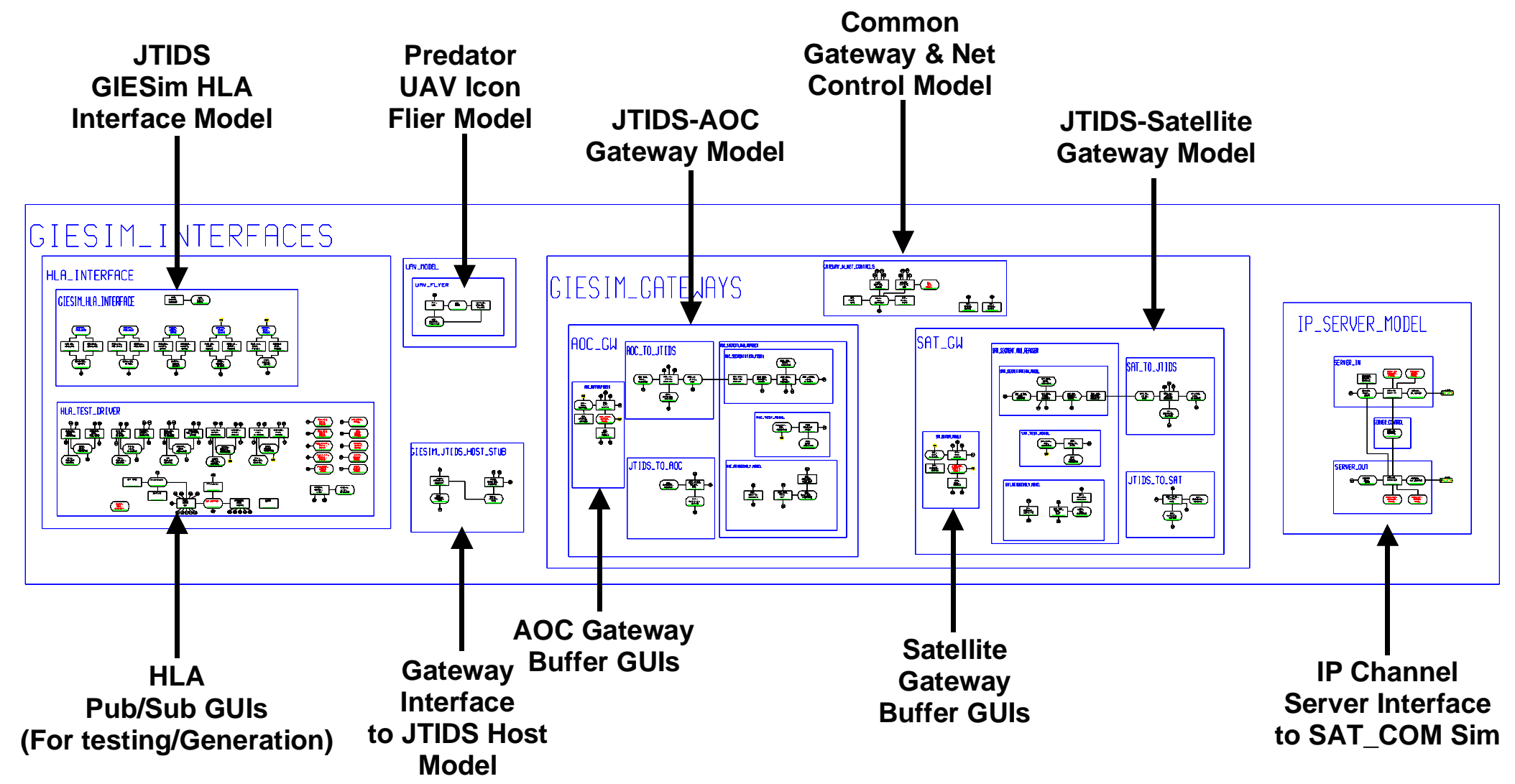

Figure 17 - Detailed Architecture of GIESim Interfaces in JTIDS 


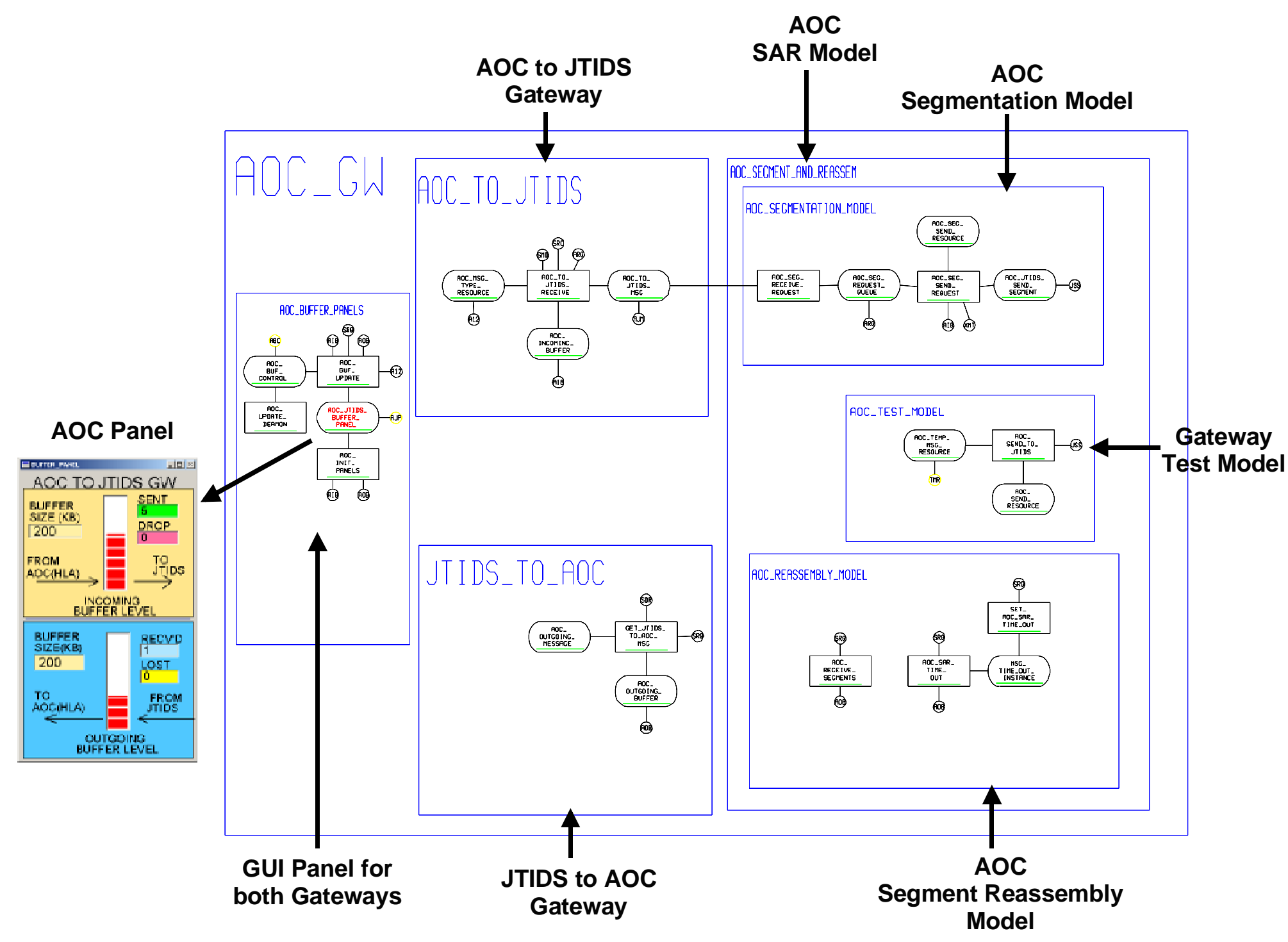

Figure 18 - AOC_GW Model in JTIDS with AOC Panel Shown 
New Processes and Changes to JTIDS Host Message Generator for GIESim

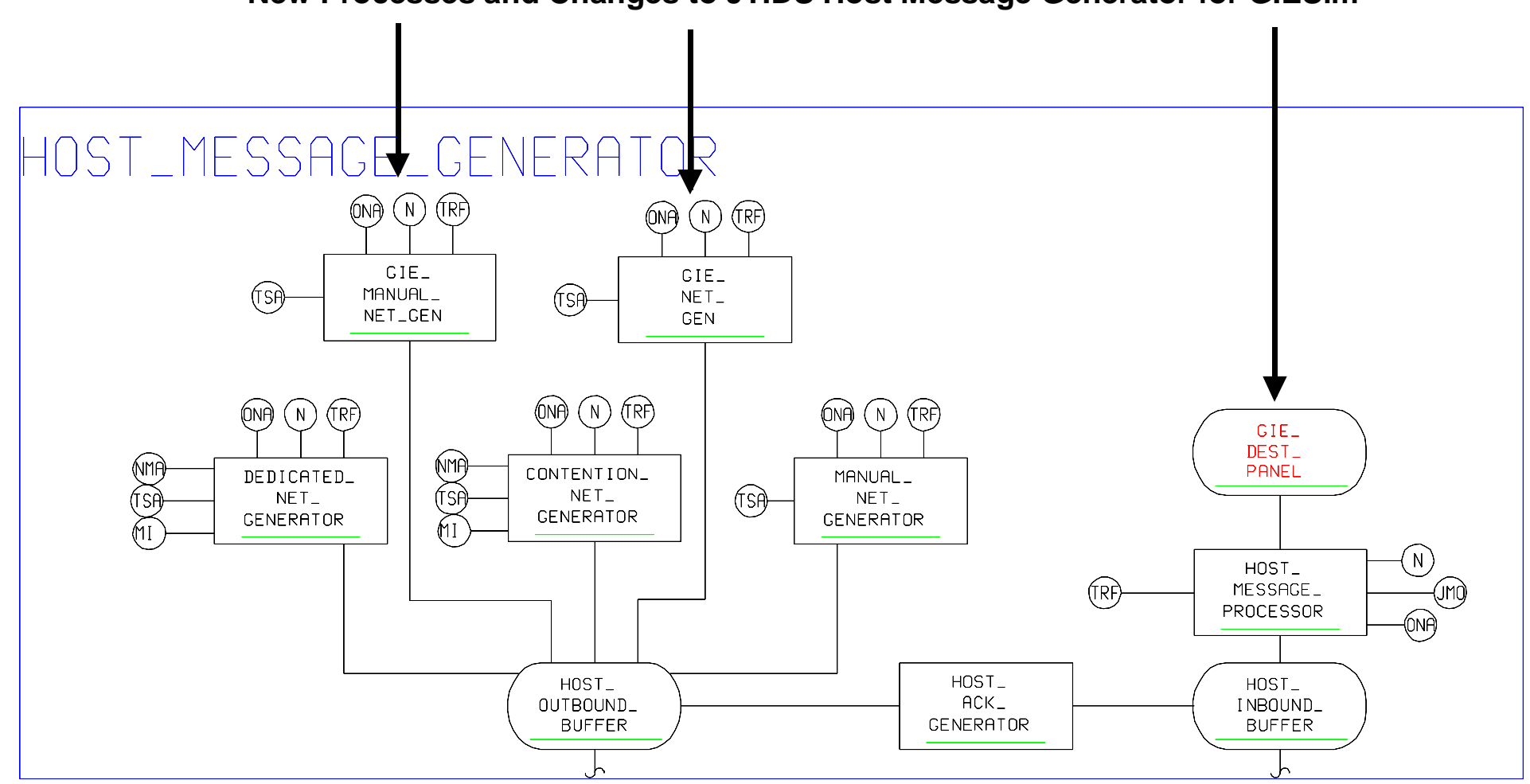

Figure 19 - JTIDS Host Model Changes for GIESim 


\section{A.3 GSS SAT_COM}

PSI proposed the addition of satellites into the DSMD through the use of our SAT_COM simulation. Satellites are important components of operational scenarios, and a communications capability used by DTIG and other systems. PSI also offered to interface SAT_COM to the Satellite Tool Kit (STK) from AGI to obtain satellite orbits and connectivity data. As stated earlier in this Final Report, this work was completed successfully.

Figure 20 shows the overall architecture of the SAT_COM simulation and indicates the models added to support the DMSD. SAT_COM is based on GSS and uses RTG for visualization of connectivity, satellites, political borders, and terrain. SAT_COM uses a top-down hierarchical model approach to simulation design along the lines discussed in the preceding section on JTIDS.

Figure 21 shows a more detailed view of the models added to SAT_COM for the GIESim DMSD. SAT_COM has an HLA interface to communicate with the other non-GSS simulations in the DMSD. This is the same HLA model hierarchy that was developed in the TEST_DRV simulation, and was imported into SAT_COM. Only minor tailoring of a couple of process calls was needed to integrate the HLA interface into SAT_COM.

Also shown in Figure 21 is the CLIENT_MODULE that supports two IP Client interfaces to the JTIDS IP Server interface. This module uses the GSS Channel Resource capability that makes the use of IP sockets, i.e., channels, very easy to use. One channel handles messages from JTIDS, and another handles message to JTIDS. In this case, the "messages" are the GIESim HLA Interactions bundled into a data attribute or block with a message type header to distinguish which HLA Interaction is being exchanged.

The SATELLITE_AND_GWS model contains relatively simple models of the Ground Stations located in Korea and the CONUS. A "bent-pipe” satellite model sends messages received on the up-links to the corresponding down-links. Messages coming from JTIDS arrive over the IP Interface and go to the Korea Ground Station and are sent to the CONUS Ground Station via the bent-pipe model. At the CONUS the message latency is computed and added to the HLA Interaction that is published. Messages sent to SAT_COM via HLA enter the CONUS Ground Station gateway and flow to the JTIDS simulation in the reverse direction. GUI Panels associated with each gateway indicate message activity.

The interface to STK required the creation of an STK_CONTROL model, which is shown in Figure 22. STK acts as an IP Server by virtue of their CONNECT interface. The SAT_COM STK_CONTROL makes an IP connection to the STK CONNECT interface and then controls STK to obtain satellite orbit data and connectivity data. Due to the non-interactive nature of STK, PSI had to manage a bulk download of data from STK which was stored in a number of local files, and uses these files to interactively access data and subsequently direct STK to update their display based on the local time. 
GSS SAT_COM Additions for GIESim SAB Demo

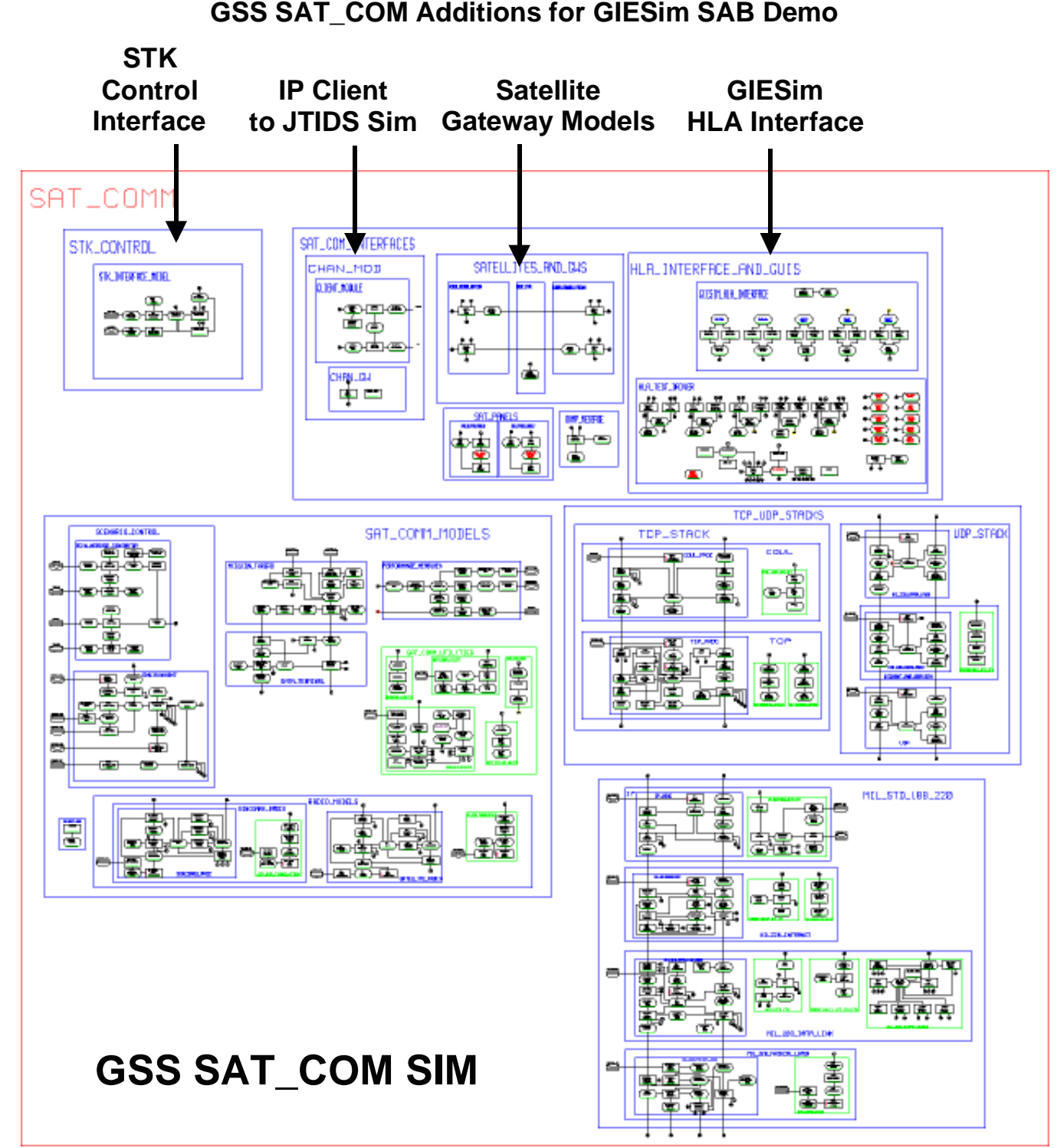

Figure 20 - GSS SAT_COM Architecture 


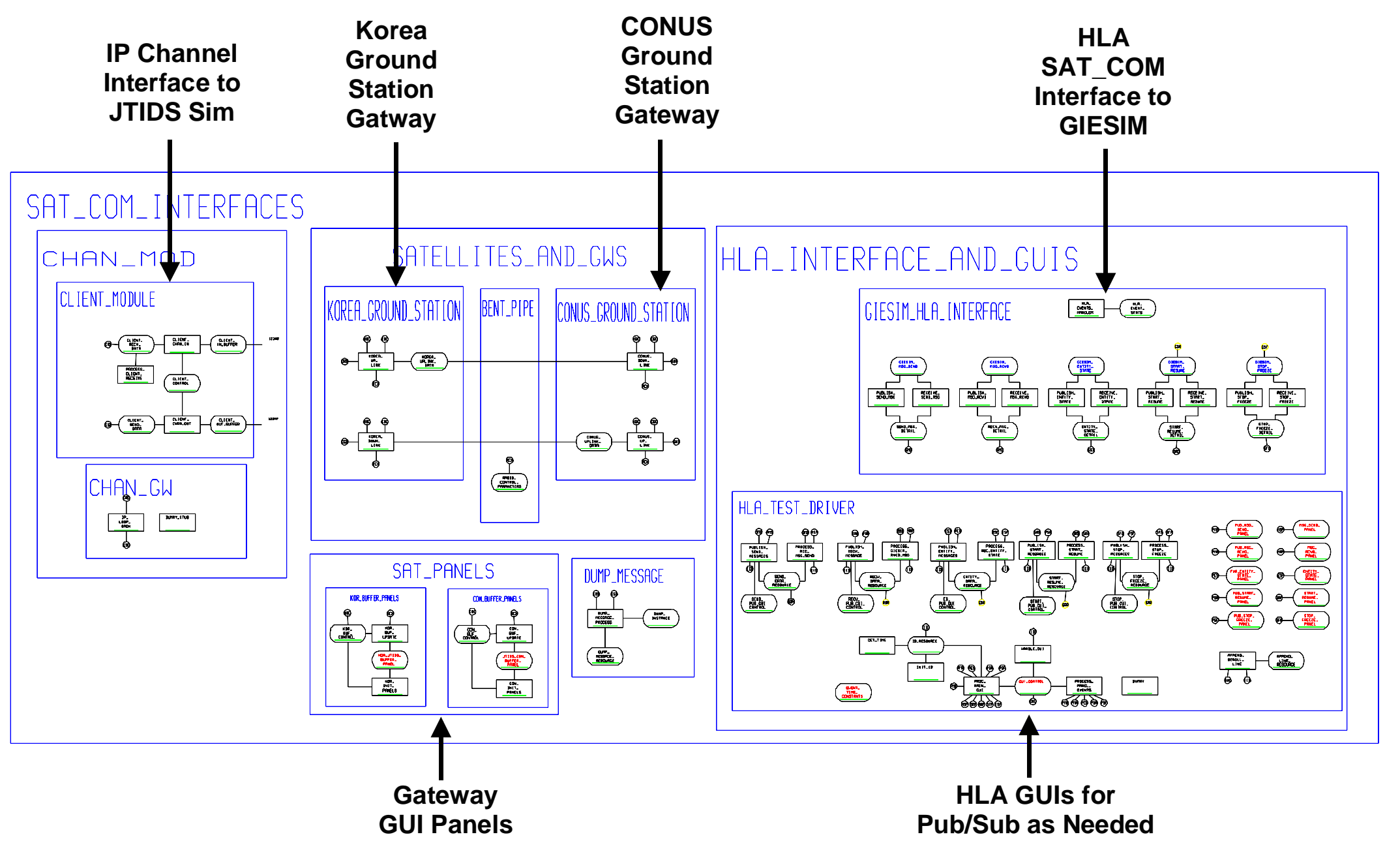

Figure 21 - Detailed Models added to SAT_COM for GIESim 


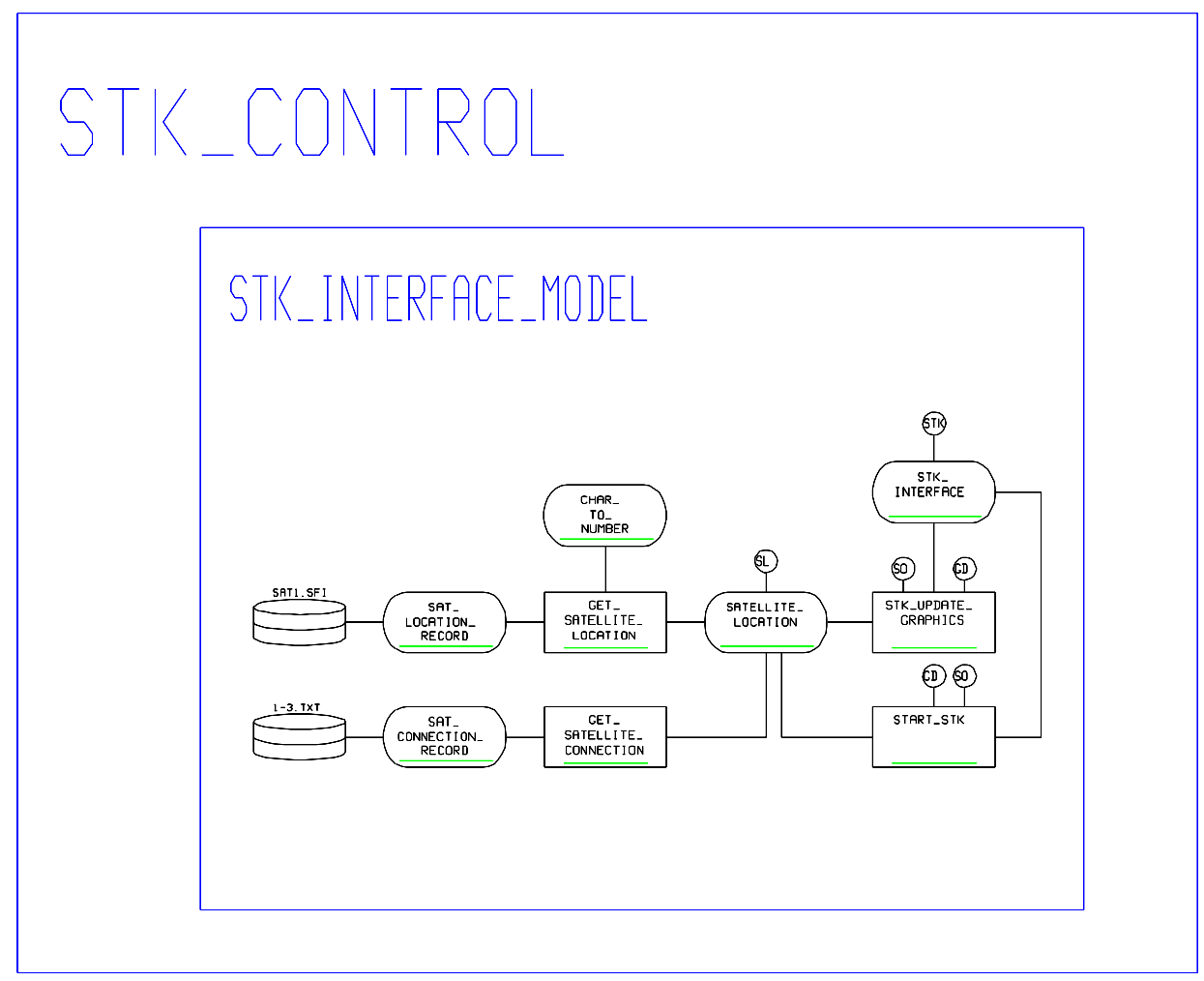

Figure 22 - SAT_COM STK Interface Control Model 